\title{
UNIFORM NULL CONTROLLABILITY FOR PARABOLIC EQUATIONS WITH DISCONTINUOUS DIFFUSION COEFFICIENTS*
}

\author{
JÉrÉmi Dardé ${ }^{1}$, Sylvain ERVedozA $^{2, * *}$ And Roberto Morales ${ }^{3}$
}

\begin{abstract}
In this article, we study the null-controllability of a heat equation in a domain composed of two media of different constant conductivities. In particular, we are interested in the behavior of the system when the conductivity of the medium on which the control does not act goes to infinity, corresponding at the limit to a perfectly conductive medium. In that case, and under suitable geometric conditions, we obtain a uniform null-controllability result. Our strategy is based on the analysis of the controllability of the corresponding wave operators and the transmutation technique, which explains the geometric conditions.
\end{abstract}

Dedicated to Enrique Zuazua with friendship.

Mathematics Subject Classification. 35K10, 35L10, 93B05, 93B07, 93B17.

Received October 26, 2020. Accepted June 7, 2021.

\section{INTRODUCTION}

\subsection{Main results}

Let $\Omega$ be a smooth $\left(\mathscr{C}^{2}\right)$ bounded domain in $\mathbb{R}^{d}\left(d \in \mathbb{N}^{*}\right), \Omega_{1}$ be a smooth strict subdomain of $\Omega$, and set $\Omega_{2}=\Omega \backslash \bar{\Omega}_{1}$.

For $\sigma_{1}>0$ and $\sigma_{2}>0$, we introduce the conductivity $\sigma=\sigma(x)$ given by

$$
\sigma(x)= \begin{cases}\sigma_{1} & \text { if } x \in \Omega_{1}, \\ \sigma_{2} & \text { if } x \in \Omega_{2},\end{cases}
$$

\footnotetext{
* The second author has been supported by the Agence Nationale de la Recherche, Project IFSMACS, grant ANR-15-CE40-0010. The first and second authors have been supported by the CIMI Labex, Toulouse, France, under grant ANR-11-LABX-0040-CIMI and the MATH AmSud program ACIPDE. The third author has been supported by FONDECYT 3200830.

Keywords and phrases: Controllability, heat equation, observability, wave equation, transmutation technique.

${ }^{1}$ Institut de Mathématiques de Toulouse, UMR 5219 - Université de Toulouse, CNRS - UPS, Toulouse, France.

2 Institut de Mathématiques de Bordeaux UMR 5251, Université de Bordeaux, Bordeaux INP, CNRS, 33400 Talence, France.

3 Departamento de Matemática, Universidad Técnica Federico Santa María, Casilla 110-V, Valparaíso, Chile.

** Corresponding author: sylvain.ervedoza@math.u-bordeaux.fr
} 
and we consider the controlled heat equation

$$
\begin{cases}\partial_{t} y_{\sigma}-\operatorname{div}\left(\sigma \nabla y_{\sigma}\right)=u_{\sigma} \mathbb{1}_{\omega}, & \text { in }(0, T) \times \Omega, \\ y_{\sigma}=0, & \text { on }(0, T) \times \partial \Omega, \\ y_{\sigma}(0)=y_{0}, & \text { in } \Omega .\end{cases}
$$

Here, $y_{\sigma}$ denotes the state (for instance the temperature), and $u_{\sigma}$ denotes the control function, assumed to be acting on a non-empty open subset $\omega$ of $\Omega$.

The main objective of this paper is to analyze the uniform null controllability of (1.2) with respect to the parameter $\sigma$, i.e., we are interested in the behavior of the controls $\left(u_{\sigma}\right)_{\sigma_{1}, \sigma_{2}>0}$ as a function of the diffusive coefficient $\sigma$.

The fact that the conductivity $\sigma$ takes the form (1.1) means that the domain $\Omega$ is filled with a material made of two media, one located in $\Omega_{1}$ and of conductivity $\sigma_{1}$, the other one being located in $\Omega_{2}$ and of conductivity $\sigma_{2}$. Also note that, with $\Omega_{1} \Subset \Omega$ and $\Omega_{2}=\Omega \backslash \overline{\Omega_{1}}$, system (1.2) can alternatively be written as a transmission problem as follows:

$$
\begin{cases}\partial_{t} y_{1, \sigma}-\sigma_{1} \Delta y_{1, \sigma}=u_{1, \sigma} \mathbb{1}_{\omega \cap \Omega_{1}}, & \text { in }(0, T) \times \Omega_{1}, \\ \partial_{t} y_{2, \sigma}-\sigma_{2} \Delta y_{2, \sigma}=u_{2, \sigma} \mathbb{1}_{\omega \cap \Omega_{2}}, & \text { in }(0, T) \times \Omega_{2}, \\ y_{1, \sigma}=y_{2, \sigma}, & \text { on }(0, T) \times \partial \Omega_{1}, \\ \sigma_{1} \partial_{\nu} y_{1, \sigma}=\sigma_{2} \partial_{\nu} y_{2, \sigma}, & \text { on }(0, T) \times \partial \Omega_{1}, \\ y_{2, \sigma}=0, & \text { on }(0, T) \times \partial \Omega, \\ \left(y_{1, \sigma}, y_{2, \sigma}\right)(0)=\left(y_{0,1}, y_{0,2}\right), & \text { in } \Omega_{1} \times \Omega_{2},\end{cases}
$$

with the correspondence $y_{\sigma}(t, x)=y_{1, \sigma}(t, x) \mathbb{1}_{\Omega_{1}}(x)+y_{2, \sigma}(t, x) \mathbb{1}_{\Omega_{2}}(x)$ for $(t, x) \in(0, T) \times \Omega$. Hereafter, we define $\nu$ to be the normal to $\partial \Omega_{1}$ oriented from $\Omega_{2}$ to $\Omega_{1}$.

In this setting, we ask what happens when one of this medium is highly conductive, that is

$$
\sigma_{1} \rightarrow \infty, \quad \sigma_{2} \text { a fixed positive constant. }
$$

Our goal is to analyze precisely the behavior of the null-controllability properties of (1.2) in the limit (1.4), which, for a fixed $\sigma$ of the form (1.1), is known when $\partial \Omega_{1} \cap \partial \Omega_{2}$ is smooth and does not intersect the boundary (see $[20-22]$ ).

We will be able to give precise results only in the following geometrical situation, that we assume from now on:

(A1) $\Omega_{1} \Subset \Omega, \Omega_{2}=\Omega \backslash \overline{\Omega_{1}}$,

(A2) There exists a point $x_{0} \in \Omega_{1}$ such that

(a) The domain $\Omega_{1}$ is star shaped with respect to $x_{0}$.

(b) The set $\omega$ is such that $\omega \subset \Omega_{2}$ and there exists $\varepsilon>0$ such that

$$
\omega \supset\left\{x \in \Omega ; d\left(x, \Gamma_{0}\right) \leqslant \varepsilon\right\} \text { where } \Gamma_{0}=\left\{x \in \partial \Omega:\left(x-x_{0}\right) \cdot \nu(x)>0\right\} .
$$

The above geometrical assumptions are illustrated in Figure 1.

We point out that, under assumptions (A1) and (A2), the control is applied only in a subset of $\Omega_{2}$. More precisely, in (1.3) we have that $\omega \cap \Omega_{1}=\emptyset$ and therefore the second equation in (1.3) is controlled directly by the action of the control, while the first one is being controlled indirectly, through the transmission conditions. In other words, we can consider $u_{1, \sigma}$ to be the null function, so the control function will now be denoted only $u_{\sigma}$ instead of $\left(u_{1, \sigma}, u_{2, \sigma}\right)$ as in (1.3). (The case $\omega \subset \Omega_{1}$ will be briefly discussed in Thm. 4.3.)

Our main result is the following one, whose proof is given in Section 3. 


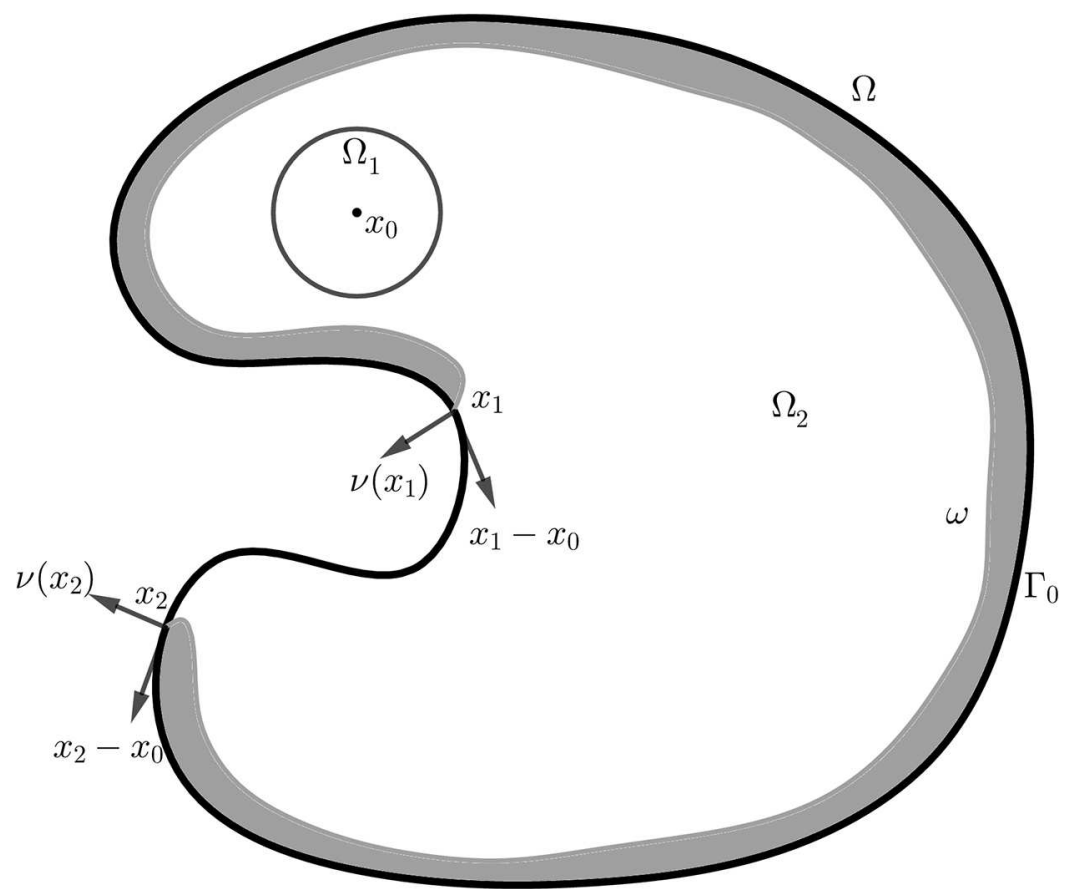

Figure 1. Geometrical situation given by (A1) and (A2), where $\omega$ is represented by the grey region.

Theorem 1.1. Let $\sigma_{2}>0$ and assume (A1) and (A2).

For all $T>0$, for all $y_{0} \in L^{2}(\Omega)$ and for all $\sigma_{1} \geqslant \sigma_{2}$, for $\sigma$ as in (1.1), there exists a control $u_{\sigma} \in L^{2}((0, T) \times$ $\omega)$ such that the solution $y_{\sigma}$ of (1.2) satisfies

$$
y_{\sigma}(T)=0 \text { in } \Omega \text {. }
$$

Moreover, the sequence of controls $\left(u_{\sigma}\right)_{\sigma_{1} \geqslant \sigma_{2}}$ is uniformly bounded, i.e. there exists a constant $C=$ $C\left(\Omega, \omega, T, \sigma_{2}\right)>0$ such that for all $\sigma_{1} \geqslant \sigma_{2}$,

$$
\left\|u_{\sigma}\right\|_{L^{2}((0, T) \times \omega)} \leqslant C\left\|y_{0}\right\|_{L^{2}(\Omega)} .
$$

Estimates (1.6) and (1.7) state the uniform null controllability of (1.2) with respect to $\sigma_{1} \geqslant \sigma_{2}$, where $\sigma_{2}>0$ is a fixed constant.

It is then natural to ask what is the limit system of (1.2) obtained as $\sigma_{1} \rightarrow \infty$ and $\sigma_{2}>0$ is fixed. This can be guessed intuitively. Indeed, since $\sigma_{1} \rightarrow \infty$, in the limit, the medium in $\Omega_{1}$ is perfectly conductive. Therefore, its temperature $y_{1}^{*}$ should be independent of the space variable. On the other hand, integrating (1.2) on $\Omega_{1}$, we see that one should have:

$$
\frac{\mathrm{d}}{\mathrm{d} t}\left(\int_{\Omega_{1}} y_{1}^{*}(t, x) \mathrm{d} x\right)=-\int_{\partial \Omega_{1}} \sigma_{2} \partial_{\nu} y_{2}^{*}(t, x) \mathrm{d} \gamma
$$

where $y_{2}^{*}$ is the temperature in the medium in $\Omega_{2}$. Besides, according to $(1.7),\left(u_{\sigma}\right)_{\sigma_{1} \geqslant \sigma_{2}}$ converges to $u^{*}$ in some sense that will be made precise in the next section. 
We should thus expect, and this will be proved afterwards (see Thm. 2.2), that the limit system of (1.2) in the limit (1.4) should be:

$$
\begin{cases}\partial_{t} y_{2}^{*}-\sigma_{2} \Delta y_{2}^{*}=u^{*} \mathbb{1}_{\omega}, & \text { in }(0, T) \times \Omega_{2}, \\ y_{2}^{*}(t, x)=0, & \text { on }(0, T) \times \partial \Omega, \\ y_{2}^{*}(t, x)=Y^{*}(t), & \text { on }(0, T) \times \partial \Omega_{1}, \\ y_{1}^{*}(t, x)=Y^{*}(t), & \text { in }(0, T) \times \Omega_{1}, \\ \partial_{t} Y^{*}+\frac{\sigma_{2}}{\left|\Omega_{1}\right|} \int_{\partial \Omega_{1}} \partial_{\nu} y_{2}^{*}(t) \mathrm{d} \gamma=0, & \text { in }(0, T), \\ y_{2}^{*}(0, \cdot)=y_{0,2}(\cdot), & \text { in } \Omega_{2}, \\ Y^{*}(0)=Y_{0}, & \end{cases}
$$

where the initial datum $Y_{0}$ is given by

$$
Y_{0}=\frac{1}{\left|\Omega_{1}\right|} \int_{\Omega_{1}} y_{0,1} \mathrm{~d} x
$$

Remark 1.2. Note that, in fact the function $y_{1}^{*}$ is now reduced to a function $Y^{*}$ depending only on the time variable, so that the system (1.8) can be thought as an equation on $\left(Y^{*}, y_{2}^{*}\right)$ only, with for all $t \in(0, T)$, $Y^{*}(t) \in \mathbb{R}$, and $y_{2}^{*}(t) \in L^{2}\left(\Omega_{2}\right)$.

Passing to the limit $\sigma_{1} \rightarrow \infty$ in Theorem 1.1, we will thus deduce the following controllability result for (1.8), see Section 3.5 for its proof.

Corollary 1.3. Let $\sigma_{2}>0$ and assume $(\boldsymbol{A 1})$ and (A2). Then for all $T>0$ and for all $\left(Y_{0}, y_{0,2}\right) \in \mathbb{R} \times L^{2}\left(\Omega_{2}\right)$ there exists a control function $u^{*} \in L^{2}((0, T) \times \omega)$ such that the solution $y^{*}$ of (1.8) satisfies

$$
y_{2}^{*}(T)=0 \text { in } \Omega_{2}, \quad \text { and } Y^{*}(T)=0 .
$$

Moreover, there exists a constant $C=C\left(\Omega, \omega, T, \sigma_{2}\right)>0$ such that

$$
\left\|u^{*}\right\|_{L^{2}((0, T) \times \omega)} \leqslant C\left\|\left(Y_{0}, y_{0,2}\right)\right\|_{\mathbb{R} \times L^{2}\left(\Omega_{2}\right)} .
$$

In addition, for $\left(Y_{0}, y_{0,2}\right) \in \mathbb{R} \times L^{2}\left(\Omega_{2}\right)$, the sequence of controls $\left(u_{\sigma}\right)_{\sigma_{1} \geqslant \sigma_{2}}$ of minimal $L^{2}\left(0, T ; L^{2}(\omega)\right)$ norm of (1.2) corresponding to an initial datum $y_{0}=Y_{0} 1_{\Omega_{1}}+y_{0,2} 1_{\Omega_{2}}$ weakly converges up to a subsequence as $\sigma_{1} \rightarrow+\infty$ to a null control $u^{*} \in L^{2}\left(0, T ; L^{2}(\omega)\right)$ for (1.8) with initial data $\left(Y_{0}, y_{0,2}\right)$.

To prove Theorem 1.1, we first remark that it is equivalent to prove a uniform observability result for the adjoint heat equation (on which we perform the change of time $t \rightarrow T-t$ as usual), that is the existence of a time $T>0$ and a constant $C=C\left(\Omega, \omega, T, \sigma_{2}\right)>0$ such that for all $\sigma_{1} \geqslant \sigma_{2}$, the solution $z_{\sigma}$ of

$$
\begin{cases}\partial_{t} z_{\sigma}-\operatorname{div}\left(\sigma \nabla z_{\sigma}\right)=0, & \text { in }(0, T) \times \Omega, \\ z_{\sigma}=0, & \text { on }(0, T) \times \partial \Omega, \\ z_{\sigma}(0)=z_{0}, & \text { in } \Omega,\end{cases}
$$

with $z_{0} \in H_{0}^{1}(\Omega)$ and $\sigma$ as in (1.1) satisfies

$$
\left\|z_{\sigma}(T)\right\|_{L^{2}(\Omega)} \leqslant C\left\|z_{\sigma}\right\|_{L^{2}((0, T) \times \omega)} .
$$


In fact, this uniform observability result will be deduced, using the transmutation technique borrowed from [11], from a uniform observability result for the corresponding wave equation:

$$
\begin{cases}\partial_{t}^{2} w_{\sigma}-\operatorname{div}\left(\sigma \nabla w_{\sigma}\right)=0, & \text { in }(0, T) \times \Omega, \\ w_{\sigma}=0, & \text { on }(0, T) \times \partial \Omega, \\ \left(w_{\sigma}, \partial_{t} w_{\sigma}\right)(0)=\left(w_{0}, w_{1}\right), & \text { in } \Omega,\end{cases}
$$

with $\left(w_{0}, w_{1}\right) \in H_{0}^{1}(\Omega) \times L^{2}(\Omega)$. This strategy is responsible for the geometric conditions (A1) and (A2), since some geometric conditions are required for the controllability of the wave equation (1.13). This is of course already the case when the velocity $\sigma$ is smooth (this is the celebrated Geometric Control Conditions of [2]) but when it is discontinuous as in (1.1), the situation is much more intricate and we refer to the recent results $[3,4,16]$.

In fact, the uniform observability result we prove for (1.13) is the following one, see Section 3.1 for its proof:

Theorem 1.4. Let $\sigma_{2}>0$ and assume Assumptions (A1) and (A2).

Then for all $T>0$ satisfying

$$
\sqrt{\sigma_{2}} T>2 \sup _{\Omega}\left\{\left|x-x_{0}\right|\right\}
$$

there exists $C>0$ such that for all $\left(w_{0}, w_{1}\right) \in H_{0}^{1}(\Omega) \times L^{2}(\Omega)$ and for all $\sigma_{1} \geqslant \sigma_{2}$, the solution $w_{\sigma}$ of (1.13) with initial datum $\left(w_{0}, w_{1}\right) \in H_{0}^{1}(\Omega) \times L^{2}(\Omega)$ and $\sigma$ as in (1.1) verifies

$$
\int_{\Omega}\left(\sigma\left|\nabla w_{0}\right|^{2}+\left|w_{1}\right|^{2}\right) \mathrm{d} x \leqslant C \int_{0}^{T} \int_{\Gamma_{0}}\left|\partial_{\nu} w\right|^{2} \mathrm{~d} \gamma \mathrm{d} t
$$

The proof of Theorem 1.4 is based on a multiplier argument. This is where the geometric conditions (A1) and (A2) appear naturally.

In fact, Theorem 1.4 has already been proved under Assumptions (A.1) and (A.2) in Chapter 6 of [24] except for the uniformity of the observability constant with respect to $\sigma_{1}$. As this is a critical argument in our proof, we will present the complete proof of Theorem 1.4 in Section 3.1 for completeness.

Remark 1.5. There are a priori several paths based on transmutation techniques to deduce Theorem 1.1 from Theorem 1.4. In particular, one could try to follow the approach in [26, 27], which would consist in first deducing from Theorem 1.4 a uniform controllability result for the wave equation, and then use a transmutation technique to deduce a uniform controllability result for the corresponding heat equation. This is in principle possible, but in our case this would be delicate since the observability estimate (1.14) implies the controllability of the corresponding wave equation with a control bounded by the norm of the initial datum in $L^{2}(\Omega) \times H^{-1}(\Omega)$. However, if we want to keep track of the dependence of the constants in terms of $\sigma$, one should be careful that the accurate norm used in $H_{0}^{1}(\Omega)$ is $\|\sqrt{\sigma} \nabla \cdot\|_{L^{2}(\Omega)}$ which depends on $\sigma$, and thus the space $H^{-1}(\Omega)$ should be endowed with the corresponding dual norm. To avoid these difficulties and follow the dependence in $\sigma$ more clearly, we have chosen to use the transmutation technique of $[11,12]$, and to avoid the use of negative Sobolev spaces.

\subsection{Related references}

Our investigations are part of the broad question of null-controllability for parabolic equations. The pioneering results for this question are the articles [15, 23], based on Carleman estimates and which apply for conductivities $\sigma \in W^{1, \infty}(\Omega)$. In our case, the conductivity given by (1.1) does not belong to $W^{1, \infty}(\Omega)$ and these results thus do not apply. 
This regularity assumption on the conductivity was then studied in details. It was proved that, in $1 \mathrm{~d}$, the heat equation (1.2) is still null-controllable for a conductivity $\sigma \in B V(\Omega)$, based on Russell's method (see e.g. [29]). The work [1] later improved this result, still in $1 \mathrm{~d}$, to the case $\sigma \in L^{\infty}(\Omega)$ using the theory of quasiconformal maps, and the more recent work [25] proposed an alternative approach for rough coefficients, still in the 1-dimensional case.

The multi-d heat equation was analysed later for piecewise $C^{2}$ conductivities with a smooth surface of $C^{0}$ discontinuity in [10] using Carleman estimates, when the control is supported in the region where the diffusion coefficient is the lowest, under some geometric conditions which are less restrictive than our assumptions (A1) and (A2). Later on, the case of $B V$ coefficients in 1d was dealt with using the Fursikov-Imanuvilov approach in [19]. The case of piecewise smooth coefficients with a smooth surface of discontinuity was then studied under no geometric assumptions in the works [5-7, 20-22], and null-controllability of (1.2) was proved in those cases.

Let us point out that, despite these numerous results, to our knowledge, the behavior of the controllability of the models (1.2) for conductivities of the form (1.1) has not been studied so far in the limit $\sigma_{1} \rightarrow \infty$, even in the $1 \mathrm{~d}$ case.

Our approach is quite different from the ones based on Carleman estimates, as it relies on the observability of the corresponding wave equation, inspired by the transmutation techniques developed in [11, 12], in a somewhat dual version of the transmutation techniques in $[26,27]$. There, the idea is to associate solutions of the wave equations to solutions of the heat equation through a time kernel, see Section 3.2 for more details.

The advantage of this technique is that our problem then reduces to the study of the observability of the corresponding wave operators, for which other techniques are available. Here, we shall follow the classical multiplier approach, introduced in [17, 24], which allows to deal with conductivities of the form (1.1) under appropriate geometric conditions, namely (A1) and (A2). These multiplier conditions are known to be very robust with respect to the regularity of the coefficients, and we refer for instance to the recent work [9] dealing with conductivities which are continuous and satisfy some suitable growth conditions in the direction of the multiplier, and to the references therein.

In fact, for waves with discontinuous conductivities, observability properties can be derived from Carleman estimates [3, 4] or microlocal analysis [16] under appropriate geometric conditions, but here again, keeping track of how it depends on the conductivity coefficients is, to our knowledge, a challenging problem.

Let us also mention that, to our knowledge, properly speaking, the controllability of the limit system (1.8) has not been dealt with in the literature, except in the 1d context in Chapter 5 of [18] where this model has been dealt with using the moment method, see also Section 4.1 for more comments. Still, the controllability result obtained in [28] on a very close system indicates that the null controllability of the limit system (1.8) can be proved directly by Carleman estimates without any geometric assumption.

\subsection{Outline}

The rest of the paper is organized as follows. Section 2 is dedicated to study the existence and uniqueness results concerning problems (1.2), (1.8) and (1.13) and their dependence with respect to $\sigma$ given by (1.1) as well as some general cases. In Section 3, the proofs of Theorem 1.1 and Corollary 1.3 are given. Finally, in Section 4 additional comments and open questions are presented.

\section{Preliminaries}

In this section, we provide several existence and uniqueness results for heat and wave equations with discontinuous diffusion / velocity coefficients of the form (1.1). We also study the limit system (1.8) and show the convergences of the solutions of (1.3) as $\sigma_{1}$ goes to infinity towards those of (1.8).

\subsection{The heat equation for general conductivities $\sigma$}

We recall the functional setting corresponding to the Cauchy problem for the parabolic equation 


$$
\begin{cases}\partial_{t} y-\operatorname{div}(\sigma \nabla y)=f, & \text { in }(0, T) \times \Omega \\ y=0, & \text { on }(0, T) \times \partial \Omega \\ y(0)=y_{0}, & \text { in } \Omega,\end{cases}
$$

for a general conductivity $\sigma$ satisfying

$$
\sigma \in L^{\infty}(\Omega) \text { and there exists } \alpha>0 \text { such that } \sigma(x) \geqslant \alpha, \quad \text { a. e. in } \Omega \text {. }
$$

We start with the definition of the bilinear form

$$
a_{\sigma}\left(y_{a}, y_{b}\right)=\int_{\Omega} \sigma \nabla y_{a} \cdot \nabla y_{b} \mathrm{~d} x
$$

for $y_{a}$ and $y_{b}$ in $H_{0}^{1}(\Omega)$. From $(2.2)$, it is clear that $a_{\sigma}$ defines a continuous bilinear form on $H_{0}^{1}(\Omega)$ which is coercive on $H_{0}^{1}(\Omega)$.

This allows to define the operator $A_{\sigma}: H_{0}^{1}(\Omega) \rightarrow H^{-1}(\Omega)$ by the formula:

$$
\forall\left(y_{a}, y_{b}\right) \in\left(H_{0}^{1}(\Omega)\right)^{2}, \quad\left\langle A_{\sigma} y_{a}, y_{b}\right\rangle_{H^{-1}(\Omega), H_{0}^{1}(\Omega)}=a_{\sigma}\left(y_{a}, y_{b}\right) .
$$

As one easily checks using Lax-Milgram theorem and condition (2.2), this operator is a self-adjoint maximal operator on $H^{-1}(\Omega)$ with domain $\mathcal{D}\left(A_{\sigma}\right)=H_{0}^{1}(\Omega)$.

Therefore, for $f \in L^{1}\left(0, T ; H^{-1}(\Omega)\right)$ and $y_{0} \in H^{-1}(\Omega)$, as a consequence of Hille-Yosida theorem, interpreting equation (2.1) as the abstract equation

$$
y^{\prime}+A_{\sigma} y=f, \quad \text { in }(0, T), \quad y(0)=y_{0},
$$

there exists a unique solution $y$ of $(2.1)$ in the class $C^{0}\left([0, T] ; H^{-1}(\Omega)\right)$.

In fact, we could also have defined the operator $\tilde{A}_{\sigma}$ on the Hilbert space $L^{2}(\Omega)$ with domain

$$
\mathcal{D}\left(\tilde{A}_{\sigma}\right)=\left\{y \in H_{0}^{1}(\Omega) \text { s.t. } \operatorname{div}(\sigma \nabla y) \in L^{2}(\Omega)\right\},
$$

and defined by

$$
\tilde{A}_{\sigma} y=-\operatorname{div}(\sigma \nabla y) \text {. }
$$

Note that $A_{\sigma}$ is the self-adjoint extension of $\tilde{A}_{\sigma}$ to $H^{-1}(\Omega)$. The only difficulty when working with $\tilde{A}_{\sigma}$ is that, without additional assumption on $\sigma$, its domain cannot be made more explicit than (2.6).

Still, one can check that $\tilde{A}_{\sigma}$ is a self-adjoint maximal operator on $L^{2}(\Omega)$. Interpreting equation $(2.1)$ as the abstract equation

$$
y^{\prime}+\tilde{A}_{\sigma} y=f, \quad \text { in }(0, T), \quad y(0)=y_{0},
$$

Hille-Yosida Theorem then yields that for any $y_{0} \in L^{2}(\Omega)$ and $f \in L^{1}\left(0, T ; L^{2}(\Omega)\right)$, the solution of (2.8) belongs to $C^{0}\left([0, T] ; L^{2}(\Omega)\right)$.

Besides, the following energy estimates can be derived: in $\mathscr{D}^{\prime}(0, T)$,

$$
\frac{\mathrm{d}}{\mathrm{d} t}\left(\frac{1}{2} \int_{\Omega}|y(t)|^{2} \mathrm{~d} x\right)+\int_{\Omega} \sigma|\nabla y(t)|^{2} \mathrm{~d} x=\int_{\Omega} f(t) y(t) \mathrm{d} x,
$$


and

$$
\frac{\mathrm{d}}{\mathrm{d} t}\left(\int_{\Omega} \sigma|\nabla y(t)|^{2} \mathrm{~d} x\right)+\int_{\Omega}\left(\left|\partial_{t} y(t)\right|^{2}+|\operatorname{div}(\sigma \nabla y(t))|^{2}\right) \mathrm{d} x=\int_{\Omega}|f(t)|^{2} \mathrm{~d} x
$$

In particular, for all time $T>0$, there exists a constant $C$ independent of $\sigma$ such that, if $y_{0} \in L^{2}(\Omega)$ and $f \in L^{2}\left(0, T ; L^{2}(\Omega)\right)$,

$$
\int_{0}^{T} \int_{\Omega} t\left(\left|\partial_{t} y(t)\right|^{2}+|\operatorname{div}(\sigma \nabla y(t))|^{2}\right) \mathrm{d} x \mathrm{~d} t \leqslant C \int_{\Omega}\left|y_{0}\right|^{2} \mathrm{~d} x+C \int_{0}^{T} \int_{\Omega}|f|^{2} \mathrm{~d} x \mathrm{~d} t .
$$

Let us finally indicate that the solution $y$ of (2.8) can also be characterized as the unique element of $L^{2}\left(0, T ; L^{2}(\Omega)\right)$ such that for all $g \in L^{2}\left(0, T ; L^{2}(\Omega)\right)$,

$$
\int_{0}^{T} \int_{\Omega} y g \mathrm{~d} x \mathrm{~d} t=\int_{0}^{T} \int_{\Omega} f z \mathrm{~d} x \mathrm{~d} t+\int_{\Omega} y_{0} z(0) \mathrm{d} x
$$

where $z$ is the solution of $-z^{\prime}+\tilde{A}_{\sigma} z=g$ in $(0, T)$ and $z(T)=0$.

\subsection{The case $\sigma$ as in (1.1): additional regularity}

In our present geometrical configuration, that is $\Omega_{1} \Subset \Omega$ and $\Omega_{2}=\Omega \backslash \overline{\Omega_{1}}$ having smooth boundaries, and with $\sigma$ as in (1.1) with constant $\left(\sigma_{1}, \sigma_{2}\right)$, the domain of $\tilde{A}_{\sigma}$ is slightly more explicit. Indeed, we have the following regularity result:

Lemma 2.1. Let $\Omega$ be a smooth bounded domain of $\mathbb{R}^{d}, \sigma$ be as in (1.1), where $\Omega_{1} \Subset \Omega$ and $\Omega_{2}=\Omega \backslash \overline{\Omega_{1}}$ have smooth boundaries. Then, for any $f \in L^{2}(\Omega)$, the solution $y \in H_{0}^{1}(\Omega)$ of

$$
-\operatorname{div}(\sigma \nabla y)=f \text { in } \Omega, \quad y=0 \text { on } \partial \Omega,
$$

is such that $y_{1}=\mathbb{1}_{\Omega_{1}}$ y and $y_{2}=\mathbb{1}_{\Omega_{2}}$ y satisfy:

$$
y_{1} \in H^{2}\left(\Omega_{1}\right) \quad \text { and } \quad y_{2} \in H^{2}\left(\Omega_{2}\right),
$$

with

$$
\left\|\left(y_{1}, y_{2}\right)\right\|_{H^{2}\left(\Omega_{1}\right) \times H^{2}\left(\Omega_{2}\right)} \leqslant C\|f\|_{L^{2}(\Omega)},
$$

for a constant $C>0$ depending on $\left(\sigma_{1}, \sigma_{2}\right)$.

Proof. This result is folklore in the literature, so we only briefly sketch it. Of course, the only difficulty is close to the interface $\partial \Omega_{1}$. The idea there is to work as in the proof of elliptic regularity close to the boundary (see e.g. [13], p. 317), that is:

- Flatten the interface by a change of variables,

- Multiply the equations by divided differences which are approximations of the second order derivatives of the solution in the tangential variables, to deduce that the second order derivatives of the solution in the tangential variables belong to $L^{2}(\Omega)$,

- Recover estimates on the second order derivatives in the normal variables from the equation directly.

Details are left to the reader. 
As a consequence, when $\sigma$ is of the form (1.1), the domain of the operator $\tilde{A}_{\sigma}$ defined on the Hilbert space $L^{2}(\Omega)$ is given as follows:

$$
\mathcal{D}\left(\tilde{A}_{\sigma}\right)=\left\{y \in H_{0}^{1}(\Omega) \text { s.t. } y_{j}=\mathbb{1}_{\Omega_{j}} y \in H^{2}\left(\Omega_{j}\right), j=1,2 \text {, with } \sigma_{1} \partial_{\nu} y_{1}=\sigma_{2} \partial_{\nu} y_{2} \text { on } \partial \Omega_{1}\right\} .
$$

\subsection{The limit system (1.8)}

In this section, we focus on the analysis of the Cauchy problem for (1.8), in which for simplicity of notation, we remove the $*$ superscript.

$$
\begin{cases}\partial_{t} y_{2}-\sigma_{2} \Delta y_{2}=f_{2}, & \text { in }(0, T) \times \Omega_{2}, \\ y_{2}(t, x)=0, & \text { on }(0, T) \times \partial \Omega, \\ y_{2}(t, x)=Y(t), & \text { on }(0, T) \times \partial \Omega_{1}, \\ \partial_{t} Y+\frac{1}{\left|\Omega_{1}\right|} \int_{\partial \Omega_{1}} \sigma_{2} \partial_{\nu} y_{2}(t) \mathrm{d} \gamma=F, & \text { in }(0, T), \\ y_{2}(0, \cdot)=y_{0,2}(\cdot), & \text { in } \Omega_{2}, \\ Y(0, \cdot)=Y_{0} & \end{cases}
$$

As before, we can define the bilinear form

$$
a_{*, \sigma_{2}}=\left(\left(Y_{a}, y_{2, a}\right),\left(Y_{b}, y_{2, b}\right)\right)=\int_{\Omega_{2}} \sigma_{2} \nabla y_{2, a} \cdot \nabla y_{2, b} \mathrm{~d} x
$$

on the set $V_{*}=\left\{\left(Y, y_{2}\right) \in \mathbb{R} \times H^{1}\left(\Omega_{2}\right)\right.$, with $\left(y_{2}\right)_{\mid \partial \Omega}=0$ and $\left.\left(y_{2}\right)_{\mid \partial \Omega_{1}}=Y\right\}$. We can thus define the operator $A_{*, \sigma_{2}}$ from $V_{*}$ to $V_{*}^{\prime}$ by

$$
\forall\left(\left(Y_{a}, y_{2, a}\right),\left(Y_{b}, y_{2, b}\right)\right) \in V_{*}^{2}, \quad\left\langle A_{\sigma}\left(Y_{a}, y_{2, a}\right),\left(Y_{b}, y_{2, b}\right)\right\rangle_{V_{*}^{\prime}, V_{*}}=a_{\sigma}\left(y_{2, a}, y_{2, b}\right) .
$$

As one easily checks using Lax-Milgram theorem and condition (2.2), this operator is a self-adjoint maximal operator on $V_{*}^{\prime}$ with domain $V_{*}$.

As before, for $\left(F, f_{2}\right) \in L^{1}\left(0, T ; V_{*}^{\prime}\right)$ and $\left(Y_{0}, y_{2,0}\right) \in V_{*}^{\prime}$, as a consequence of Hille-Yosida theorem, interpreting equation (2.15) as the abstract equation

$$
\left(Y, y_{2}\right)^{\prime}+A_{*, \sigma_{2}}\left(Y, y_{2}\right)=\left(F, f_{2}\right), \quad \text { in }(0, T), \quad\left(Y, y_{2}(0)\right)=\left(Y_{0}, y_{2,0}\right)
$$

there exists a unique solution $\left(Y, y_{2}\right)$ of $(2.15)$ in the class $C^{0}\left([0, T] ; V_{*}^{\prime}\right)$.

But the space $V_{*}^{\prime}$ is not easy to deal with, and we prefer to give a functional setting based on the usual $L^{2}$ space. We thus introduce the space

$$
H_{*}=\mathbb{R} \times L^{2}\left(\Omega_{2}\right)
$$

which, endowed with the scalar product,

$$
\left(\left(Y, y_{2}\right),\left(\tilde{Y}, \tilde{y}_{2}\right)\right)_{H_{*}}=\left|\Omega_{1}\right| Y \tilde{Y}+\int_{\Omega_{2}} y_{2} \tilde{y}_{2} \mathrm{~d} x .
$$

is a Hilbert space. We then introduce the operator $\tilde{A}_{*, \sigma_{2}}$ defined on $H_{*}$ with domain

$$
\mathcal{D}\left(\tilde{A}_{*, \sigma_{2}}\right)=\left\{\left(Y, y_{2}\right) \in V_{*} \text { s.t. } y_{2} \in H^{2}\left(\Omega_{2}\right)\right\},
$$


and defined for $\left(Y, y_{2}\right) \in \mathcal{D}\left(\tilde{A}_{*, \sigma_{2}}\right)$ by

$$
\tilde{A}_{*, \sigma_{2}}\left(Y, y_{2}\right)=\left(\frac{\sigma_{2}}{\left|\Omega_{1}\right|} \int_{\partial \Omega_{2}} \partial_{\nu} y_{2} \mathrm{~d} \gamma,-\sigma_{2} \Delta y_{2}\right)
$$

One can check that $\tilde{A}_{*, \sigma_{2}}$ is a self-adjoint maximal operator on $H_{*}$. Interpreting equation (2.15) as the abstract equation

$$
\left(Y, y_{2}\right)^{\prime}+\tilde{A}_{*, \sigma_{2}}\left(Y, y_{2}\right)=\left(F, f_{2}\right), \quad \text { in }(0, T), \quad\left(Y, y_{2}\right)(0)=\left(Y_{0}, y_{2,0}\right)
$$

Hille-Yosida Theorem then yields that for any $\left(Y_{0}, y_{2,0}\right) \in H_{*}$ and $\left(F, f_{2}\right) \in L^{1}\left(0, T ; H_{*}\right)$, the solution of $(2.19)$ belongs to $C^{0}\left([0, T] ; H_{*}\right)$.

Besides, the following energy estimates can be derived: in $\mathscr{D}^{\prime}(0, T)$,

$$
\frac{1}{2} \frac{\mathrm{d}}{\mathrm{d} t}\left(\left|\Omega_{1}\right||Y(t)|^{2}+\int_{\Omega_{2}}\left|y_{2}(t)\right|^{2} \mathrm{~d} x\right)+\int_{\Omega_{2}} \sigma_{2}\left|\nabla y_{2}(t)\right|^{2} \mathrm{~d} x=\left|\Omega_{1}\right| F(t) Y(t)+\int_{\Omega} f_{2}(t) y_{2}(t) \mathrm{d} x .
$$

Let us finally indicate that the solution $\left(Y, y_{2}\right)$ of $(2.19)$ can be interpreted as the unique element of $L^{2}\left(0, T ; H_{*}\right)$ such that for all $\left(G, g_{2}\right) \in L^{2}\left(0, T ; H_{*}\right)$,

$$
\left|\Omega_{1}\right| \int_{0}^{T} Y G \mathrm{~d} t+\int_{0}^{T} \int_{\Omega} y_{2} g_{2} \mathrm{~d} x \mathrm{~d} t=\left|\Omega_{1}\right| \int_{0}^{T} F Z \mathrm{~d} t+\int_{0}^{T} \int_{\Omega} f_{2} z_{2} \mathrm{~d} x \mathrm{~d} t+\left|\Omega_{1}\right| Y_{0} Z(0)+\int_{\Omega} y_{0,2} z_{2}(0) \mathrm{d} x
$$

where $\left(Z, z_{2}\right)$ is the solution of $-\left(Z, z_{2}\right)^{\prime}+\tilde{A}_{\sigma}\left(Z, z_{2}\right)=\left(G, g_{2}\right)$ in $(0, T)$ and $\left(Z, z_{2}\right)(T)=0$.

\subsection{A convergence result}

In this section, we explain how to pass to the limit in systems (1.2) to derive equation (1.8).

In all this section, $\sigma_{2}>0$ is fixed and $\sigma_{1} \geqslant 1$ is going to infinity. Since the conductivity $\sigma$ in (1.1) is characterized by $\left(\sigma_{1}, \sigma_{2}\right)$, we will write the sequence of functions associated to the conductivity $\sigma$ as $\left(f_{\sigma}\right)_{\sigma_{1} \geqslant 1}$.

Theorem 2.2. Let $\sigma_{2}>0$ be fixed, $T>0, y_{0} \in L^{2}(\Omega)$, and let $\left(f_{\sigma}\right)_{\sigma_{1} \geqslant 1}$ be a sequence of $L^{2}\left(0, T ; L^{2}(\Omega)\right)$, which weakly converges as $\sigma_{1} \rightarrow \infty$ to $f^{*}$ in $L^{2}\left(0, T ; L^{2}(\Omega)\right)$.

For $\sigma_{1} \geqslant 1$, let $\left(y_{\sigma}\right)_{\sigma_{1} \geqslant 1}$ be the solution of

$$
\begin{cases}\partial_{t} y_{1, \sigma}-\sigma_{1} \Delta y_{1, \sigma}=f_{1, \sigma} \mathbb{1}_{\Omega_{1}}, & \text { in }(0, T) \times \Omega_{1}, \\ \partial_{t} y_{2, \sigma}-\sigma_{2} \Delta y_{2, \sigma}=f_{2, \sigma} \mathbb{1}_{\Omega_{2}}, & \text { in }(0, T) \times \Omega_{2}, \\ y_{1, \sigma}=y_{2, \sigma}, & \text { on }(0, T) \times \partial \Omega_{1}, \\ \sigma_{1} \partial_{\nu} y_{1, \sigma}=\sigma_{2} \partial_{\nu} y_{2, \sigma}, & \text { on }(0, T) \times \partial \Omega_{1}, \\ y_{2, \sigma}=0, & \text { in }(0, T) \times \partial \Omega, \\ \left(y_{1, \sigma}, y_{2, \sigma}\right)(0)=\left(y_{0,1}, y_{0,2}\right), & \text { in } \Omega_{1} \times \Omega_{2},\end{cases}
$$

where $y_{0,1}=\left.y_{0}\right|_{\Omega_{1}}$ and $y_{0,2}=\left.y_{0}\right|_{\Omega_{2}}$. Let us also define

$$
Y_{0}=\frac{1}{\left|\Omega_{1}\right|} \int_{\Omega_{1}} y_{0,1}(x) \mathrm{d} x, \text { and }\left(F^{*}(t), f_{2}^{*}(t, x)\right)=\left(\frac{1}{\left|\Omega_{1}\right|} \int_{\Omega_{1}} f^{*}(t, x) \mathrm{d} x,\left.f^{*}\right|_{\Omega_{2}}(t, x)\right),
$$


a.e. in $t \in(0, T), x \in \Omega_{2}$, and $\left(Y^{*}, y_{2}^{*}\right)$ given by

$$
\begin{cases}\partial_{t} y_{2}^{*}-\sigma_{2} \Delta y_{2}^{*}=f_{2}^{*}, & \text { in }(0, T) \times \Omega_{2}, \\ y_{2}^{*}(t, x)=0, & \text { on }(0, T) \times \partial \Omega, \\ y_{2}^{*}(t, x)=Y^{*}(t), & \text { on }(0, T) \times \partial \Omega_{1}, \\ \partial_{t} Y^{*}+\frac{\sigma_{2}}{\left|\Omega_{1}\right|} \int_{\partial \Omega_{1}} \partial_{\nu} y_{2}(t) \mathrm{d} \gamma=F^{*}, & \text { in }(0, T), \\ y_{2}^{*}(0, \cdot)=y_{0,2}(\cdot), & \text { in } \Omega_{2}, \\ Y^{*}(0, \cdot)=Y_{0} . & \end{cases}
$$

Then the sequence $\left(y_{\sigma}\right)_{\sigma_{1} \geqslant 1}$ weakly converges to $y^{*}=Y^{*} \mathbb{1}_{\Omega_{1}}+y_{2}^{*} \mathbb{1}_{\Omega_{2}}$ as $\sigma_{1} \rightarrow \infty$ in $L^{2}\left(0, T ; H_{0}^{1}(\Omega)\right)$, weakly-* in $L^{\infty}\left(0, T ; L^{2}(\Omega)\right)$, and strongly in $C_{\mathrm{loc}}^{0}\left((0, T] ; L^{2}(\Omega)\right)$.

Proof. First of all, as $\left(f_{\sigma}\right)_{\sigma_{1} \geqslant 1}$ weakly converges to $f^{*}$ in $L^{2}\left(0, T ; L^{2}(\Omega)\right)$ as $\sigma_{1} \rightarrow \infty$, the sequence is uniformly bounded, which easily implies from $(2.9)$ that $\left(y_{\sigma}\right)_{\sigma_{1} \geqslant 1}$ is bounded in $L^{\infty}\left(0, T ; L^{2}(\Omega)\right) \cap L^{2}\left(0, T ; H_{0}^{1}(\Omega)\right)$ and that

$$
\int_{0}^{T} \int_{\Omega_{1}} \sigma_{1}\left|\nabla y_{1, \sigma}\right|^{2} \mathrm{~d} x \leqslant C .
$$

Therefore as $\sigma_{1} \rightarrow+\infty$, the sequence $\left(y_{\sigma}\right)_{\sigma_{1} \geqslant 1}$ weakly converges, up to a subsequence, in $L^{\infty}\left(0, T ; L^{2}(\Omega)\right) \cap$ $L^{2}\left(0, T ; H_{0}^{1}(\Omega)\right)$ toward some $y^{*}$ such that $\nabla y^{*}=0$ in $\Omega_{1}$, i.e. there exists a function $Y^{*} \in L^{2}(0, T)$ such that $y^{*} \mathbb{1}_{\Omega_{1}}=Y^{*}$ in $(0, T) \times \Omega_{1}$.

Now, we will use the following

Lemma 2.3. Let $\left(G, g_{2}\right) \in L^{2}(0, T) \times L^{2}\left(0, T ; L^{2}\left(\Omega_{2}\right)\right)$ and let $\left(Z, z_{2}\right)$ be the solution of

$$
-\left(Z, z_{2}\right)^{\prime}+\tilde{A}_{*, \sigma_{2}}\left(Z, z_{2}\right)=\left(G, g_{2}\right) \quad \text { in }(0, T), \quad\left(Z, z_{2}\right)(T)=(0,0)
$$

where $\tilde{A}_{*, \sigma_{2}}$ given in (2.17). Set then

$$
g(t, x)=G(t) \mathbb{1}_{\Omega_{1}}(x)+g_{2}(t, x) \mathbb{1}_{\Omega_{2}}(x), \quad(t, x) \in(0, T) \times \Omega,
$$

and for $\sigma_{1} \geqslant 1$, introduce $z_{\sigma}$ the solution of

$$
-z_{\sigma}^{\prime}+\tilde{A}_{\sigma} z_{\sigma}=g, \quad \text { in }(0, T), \quad z_{\sigma}(T)=0
$$

for $\sigma$ characterized by $\left(\sigma_{1}, \sigma_{2}\right)$ as in (1.1).

Then, setting

$$
z^{*}(t, x)=Z(t) \mathbb{1}_{\Omega_{1}}(x)+z_{2}(t, x) \mathbb{1}_{\Omega_{2}}(x), \quad(t, x) \in(0, T) \times \Omega,
$$

we have the following convergence result:

$$
\begin{aligned}
& z_{\sigma} \underset{\sigma_{1} \rightarrow \infty}{\longrightarrow} z^{*} \quad \text { strongly in } L^{2}\left(0, T ; L^{2}(\Omega)\right) \\
& z_{\sigma}(0, \cdot) \underset{\sigma_{1} \rightarrow \infty}{\longrightarrow} z^{*}(0, \cdot) \quad \text { strongly in } L^{2}(\Omega) .
\end{aligned}
$$

The proof of Lemma 2.3 will be given afterwards, and we first resume the proof of Theorem 2.2. 
The basic idea is to identify the limit $y^{*}$ by looking at the weak formulations (2.12) satisfied by $y_{\sigma}$ and pass to the limit in them. We thus take $\left(G, g_{2}\right) \in L^{2}(0, T) \times L^{2}\left(0, T ; L^{2}\left(\Omega_{2}\right)\right)$, and introduce $z_{\sigma}$ the corresponding solution of (2.27) for $\sigma$ characterized by $\left(\sigma_{1}, \sigma_{2}\right)$ as in (1.1). Therefore, for all $\sigma_{1} \geqslant 1$, we get

$$
\int_{0}^{T} \int_{\Omega} y_{\sigma} g \mathrm{~d} x \mathrm{~d} t=\int_{0}^{T} \int_{\Omega} f_{\sigma} z_{\sigma} \mathrm{d} x \mathrm{~d} t+\int_{\Omega} y_{0} z_{\sigma}(0) \mathrm{d} x
$$

Using the convergences (2.29) and (2.30), we can pass to the limit $\sigma_{1} \rightarrow \infty$ in these identities:

$$
\int_{0}^{T} \int_{\Omega} y^{*} g \mathrm{~d} x \mathrm{~d} t=\int_{0}^{T} \int_{\Omega} f^{*} z^{*} \mathrm{~d} x \mathrm{~d} t+\int_{\Omega} y_{0} z^{*}(0) \mathrm{d} x .
$$

where $z^{*}$ is given by $(2.28)$.

We then use the facts that $\left.y^{*}\right|_{\Omega_{1}}=Y^{*}$ in $(0, T) \times \Omega_{1}$, that $g$ is constant in $\Omega_{1}$ equal to $G$, that $\left.z^{*}\right|_{\Omega_{1}}=Z$ in $(0, T) \times \Omega_{1}$, where $Z$ depends only on the time variable, and that $z^{*}(0)$ is constant, equal to $Z(0)$ in $\Omega_{1}$.

Accordingly, we have

$$
\begin{aligned}
& \int_{0}^{T} \int_{\Omega} y^{*} g \mathrm{~d} x \mathrm{~d} t=\int_{0}^{T}\left|\Omega_{1}\right| Y^{*}(t) G(t) \mathrm{d} t+\int_{0}^{T} \int_{\Omega_{2}} y_{2}^{*} g_{2} \mathrm{~d} x \mathrm{~d} t \\
& \int_{0}^{T} \int_{\Omega} f^{*} z^{*} \mathrm{~d} x \mathrm{~d} t=\int_{0}^{T}\left(\int_{\Omega_{1}} f^{*} \mathrm{~d} x\right) Z \mathrm{~d} t+\int_{0}^{T} \int_{\Omega_{2}} f^{*} z_{2} \mathrm{~d} x \mathrm{~d} t \\
& \int_{\Omega} y_{0} z^{*}(0) \mathrm{d} x=\left(\int_{\Omega_{1}} y_{0} \mathrm{~d} x\right) Z(0)+\int_{\Omega_{2}} y_{0} z_{2}(0, \cdot) \mathrm{d} x .
\end{aligned}
$$

Therefore, identity $(2.31)$ can be rewritten as follows: for all $\left(G, g_{2}\right) \in L^{2}(0, T) \times L^{2}\left(0, T ; L^{2}\left(\Omega_{2}\right)\right)$, denoting by $\left(Z, z_{2}\right)$ the solution of $(2.25)$, we have

$$
\begin{aligned}
\int_{0}^{T}\left|\Omega_{1}\right| Y^{*}(t) G(t) \mathrm{d} t+\int_{0}^{T} \int_{\Omega_{2}} y_{2}^{*} g_{2} \mathrm{~d} x \mathrm{~d} t= & \int_{0}^{T}\left(\int_{\Omega_{1}} f^{*} \mathrm{~d} x\right) Z \mathrm{~d} t+\int_{0}^{T} \int_{\Omega_{2}} f^{*} z_{2} \mathrm{~d} x \mathrm{~d} t \\
& +\left(\int_{\Omega_{1}} y_{0} \mathrm{~d} x\right) Z(0)+\int_{\Omega_{2}} y_{0,2} z_{2}(0, \cdot) \mathrm{d} x .
\end{aligned}
$$

According to (2.21), this identity shows that $\left(Y^{*}, y_{2}^{*}\right)$ is indeed the solution of $(2.24)$. By uniqueness of the solutions of $(2.24)$, the whole sequence $\left(y_{\sigma}\right)_{\sigma_{1} \geqslant 1}$ weakly converges to $y^{*}=y_{1}^{*} \mathbb{1}_{\Omega_{1}}+y_{2}^{*} \mathbb{1}_{\Omega_{2}}$.

To finish the proof of Theorem 2.2, we only have to check the convergences in the mentioned functional settings. The fact that the sequence $\left(y_{\sigma}\right)_{\sigma_{1} \geqslant 1}$ weakly converges to $y^{*}=y_{1}^{*} \mathbb{1}_{\Omega_{1}}+y_{2}^{*} \mathbb{1}_{\Omega_{2}}$ as $\sigma_{1} \rightarrow \infty$ in $L^{2}\left(0, T ; H_{0}^{1}(\Omega)\right)$ has already been done. The weak-* convergence in $L^{\infty}\left(0, T ; L^{2}(\Omega)\right)$ follows from the uniform boundedness of $\left(y_{\sigma}\right)_{\sigma_{1} \geqslant 1}$ in $L^{\infty}\left(0, T ; L^{2}(\Omega)\right)$.

To get the strong convergence of the sequence $\left(y_{\sigma}\right)_{\sigma_{1} \geqslant 1}$ in $C_{\mathrm{loc}}^{0}\left((0, T] ; L^{2}(\Omega)\right)$, we simply use the uniform bound given by (2.11), which gives that, for all $\tau_{0} \in(0, T)$ the sequence $\left(y_{\sigma}\right)_{\sigma_{1} \geqslant 1}$ is bounded in $H^{1}\left(\tau_{0}, T ; L^{2}(\Omega)\right)$. Therefore, since $\left(y_{\sigma}\right)_{\sigma_{1} \geqslant 1}$ is also bounded in $L^{2}\left(0, T ; H_{0}^{1}(\Omega)\right)$ from $(2.9)$, by Aubin-Lions' theorem, the sequence $\left(y_{\sigma}\right)_{\sigma_{1} \geqslant 1}$ strongly converges in $C^{0}\left(\left(\tau_{0}, T\right] ; L^{2}(\Omega)\right)$ as $\sigma_{1} \rightarrow \infty$. This concludes the proof of Theorem 2.2 up to the proof of Lemma 2.3 done afterwards.

Proof of Lemma 2.3. Let $\left(G, g_{2}\right) \in L^{2}(0, T) \times L^{2}\left(0, T ; L^{2}\left(\Omega_{2}\right)\right)$ and let $\left(Z, z_{2}\right)$ be the solution of $(2.25)$.

Then define the function $g$ in $(0, T) \times \Omega$ by formula (2.26), and introduce the solution $z_{\sigma}$ of $(2.27)$. According to $(2.10)$ (applied to $z_{\sigma}(T-t)$, which satisfies $(2.8)$ ), we get that for all $\sigma_{1} \geqslant 1$, 


$$
z_{\sigma} \in L^{2}\left(0, T ; \mathcal{D}\left(\tilde{A}_{\sigma}\right)\right) \cap L^{\infty}\left(0, T ; H^{1}(\Omega)\right) \cap H^{1}\left(0, T ; L^{2}(\Omega)\right)
$$

with

$$
\sup _{t \in[0, T]}\left(\int_{\Omega} \sigma\left|\nabla z_{\sigma}(t)\right|^{2} \mathrm{~d} x\right)+\int_{0}^{T} \int_{\Omega}\left(\left|\partial_{t} z_{\sigma}(t)\right|^{2}+\left|\operatorname{div}\left(\sigma \nabla z_{\sigma}(t)\right)\right|^{2}\right) \mathrm{d} x \mathrm{~d} t \leqslant \int_{0}^{T} \int_{\Omega}|g(t)|^{2} \mathrm{~d} x \mathrm{~d} t .
$$

We now set $z_{1, \sigma}=\left.z_{\sigma}\right|_{\Omega_{1}}$ and $z_{2, \sigma}=\left.z_{\sigma}\right|_{\Omega_{2}}$.

According to the above regularity and Lemma 2.1, we can integrate the equation of $z_{\sigma}$ on $\Omega_{1}$ and obtain that in $\mathscr{D}^{\prime}(0, T)$ (recall that $\nu$ is the normal oriented from $\Omega_{2}$ to $\Omega_{1}$ ),

$$
-\frac{\mathrm{d}}{\mathrm{d} t}\left(\int_{\Omega_{1}} z_{1, \sigma}(t, x) \mathrm{d} x\right)+\int_{\partial \Omega_{1}} \sigma_{1} \partial_{\nu} z_{1, \sigma}(t, x) \mathrm{d} x=\int_{\Omega_{1}} g(t, x) \mathrm{d} x
$$

Therefore, using the transmission conditions and the definition of $g$, we get

$$
-\frac{\mathrm{d}}{\mathrm{d} t}\left(\int_{\Omega_{1}} z_{1, \sigma}(t, x) \mathrm{d} x\right)+\int_{\partial \Omega_{1}} \sigma_{2} \partial_{\nu} z_{2, \sigma}(t, x) \mathrm{d} x=\int_{\Omega_{1}} g(t, x) \mathrm{d} x=\left|\Omega_{1}\right| G(t) .
$$

Besides, $\left(z_{1, \sigma}\right)_{\sigma_{1} \geqslant 1}$ is bounded in $L^{\infty}\left(0, T ; H^{1}\left(\Omega_{1}\right)\right) \cap H^{1}\left(0, T ; L^{2}\left(\Omega_{1}\right)\right)$ with $\nabla z_{1, \sigma}$ going to 0 strongly in $L^{\infty}\left(0, T ; L^{2}\left(\Omega_{1}\right)\right) \cap L^{2}\left(0, T ; H^{1}\left(\Omega_{1}\right)\right)$ as $\sigma_{1} \rightarrow \infty$, so that $\left(z_{1, \sigma}\right)_{\sigma_{1} \geqslant 1}$ weakly converges as $\sigma_{1} \rightarrow \infty$ (up to subsequence) to $z_{1}^{*}$ in $L^{2}\left(0, T ; H^{1}\left(\Omega_{1}\right)\right) \cap H^{1}\left(0, T ; L^{2}\left(\Omega_{1}\right)\right)$ satisfying $\nabla z_{1}^{*}=0$ in $(0, T) \times \Omega_{1}$ and $z_{1}^{*}(T)=0$.

Moreover, by (2.9)-(2.10), $\left(z_{2, \sigma}\right)_{\sigma_{1} \geqslant 1}$ is bounded in $L^{\infty}\left(0, T ; H^{1}\left(\Omega_{2}\right)\right) \cap H^{1}\left(0, T ; L^{2}\left(\Omega_{2}\right)\right)$. The sequence $\left(z_{2, \sigma}\right)_{\sigma_{1} \geqslant 1}$ thus weakly converges to some $z_{2}^{*}$ in $L^{2}\left(0, T ; H^{1}\left(\Omega_{2}\right)\right) \cap H^{1}\left(0, T ; L^{2}\left(\Omega_{2}\right)\right)$ as $\sigma_{1} \rightarrow \infty$ satisfying $z_{2}^{*}(T)=0$. Besides, $\left(\operatorname{div}\left(\sigma_{2} \nabla z_{2, \sigma}\right)\right)_{\sigma_{1} \geqslant 1}$ is bounded in $L^{2}\left(0, T ; L^{2}\left(\Omega_{2}\right)\right)$ from $(2.10)$, and thus $\left(\sigma_{2} \partial_{\nu} z_{2, \sigma}\right)_{\sigma_{1} \geqslant 1}$ weakly converges as $\sigma_{1} \rightarrow \infty$ to $\sigma_{2} \partial_{\nu} z_{2}^{*}$ in $L^{2}\left(0, T ; L^{2}\left(\partial \Omega_{1}\right)\right)$. Furthermore, for all smooth $\varphi \in \mathscr{D}([0, T] \times \Omega)$ such that for all $t \in[0, T],\left.\varphi(t)\right|_{\partial \Omega_{1}}$ is constant,

$$
\begin{aligned}
\int_{0}^{T} \int_{\Omega_{2}} g \varphi \mathrm{d} t \mathrm{~d} x-\int_{0}^{T}\left(\int_{\partial \Omega_{1}} \sigma_{2} \partial_{\nu} z_{2, \sigma} \mathrm{d} \gamma(x)\right) & \left.\varphi(t)\right|_{\partial \Omega_{1}} \mathrm{~d} t+\int_{0}^{T} \int_{\partial \Omega_{1}} z_{2, \sigma} \sigma_{2} \partial_{\nu} \varphi \mathrm{d} \gamma \mathrm{d} t \\
& =-\int_{\Omega_{2}} z_{2, \sigma}(0, x) \varphi(0, x) \mathrm{d} x-\int_{0}^{T} \int_{\Omega_{2}} z_{2, \sigma}\left(\partial_{t} \varphi-\sigma_{2} \Delta \varphi\right) \mathrm{d} x \mathrm{~d} t
\end{aligned}
$$

and the above convergences easily give that for all smooth $\varphi \in \mathscr{D}([0, T] \times \Omega)$ such that for all $t \in[0, T],\left.\varphi(t)\right|_{\partial \Omega_{1}}$ is constant,

$$
\begin{aligned}
\int_{0}^{T} \int_{\Omega_{2}} g \varphi \mathrm{d} x \mathrm{~d} t-\left.\int_{0}^{T}\left(\int_{\partial \Omega_{2}} \sigma_{2} \partial_{\nu} z_{2}^{*} \mathrm{~d} \gamma\right) \varphi(t)\right|_{\partial \Omega_{1}} \mathrm{~d} t+\int_{0}^{T} \int_{\partial \Omega_{1}} z_{2}^{*} \sigma_{2} \partial_{\nu} \varphi \mathrm{d} \gamma \mathrm{d} t \\
=-\int_{\Omega_{2}} z_{2}^{*}(0, x) \varphi(0, x) \mathrm{d} x-\int_{0}^{T} \int_{\Omega_{2}} z_{2}^{*}\left(\partial_{t} \varphi-\sigma_{2} \Delta \varphi\right) \mathrm{d} x \mathrm{~d} t
\end{aligned}
$$

We can also pass to the limit in (2.32) and obtain that in $\mathscr{D}^{\prime}(0, T)$,

$$
-\frac{\mathrm{d}}{\mathrm{d} t}\left(\int_{\Omega_{1}} z_{1}^{*}(t) \mathrm{d} x\right)+\int_{\partial \Omega_{1}} \sigma_{2} \partial_{\nu} z_{2}^{*}(t) \mathrm{d} \gamma=\left|\Omega_{1}\right| G(t)
$$


that is, since $\nabla z_{1}^{*}=0$,

$$
-\left|\Omega_{1}\right| \frac{d z_{1}^{*}(t)}{\mathrm{d} t}+\int_{\partial \Omega_{1}} \sigma_{2} \partial_{\nu} z_{2}^{*}(t) \mathrm{d} x=\left|\Omega_{1}\right| G(t) .
$$

Finally, the above convergences also allow to pass to the limit in the identity $z_{1, \sigma}(t, x)=z_{2, \sigma}(t, x)$ on $(0, T) \times$ $\partial \Omega_{1}$ as $\sigma_{1} \rightarrow \infty$, so that we also have

$$
z_{1}^{*}(t)=z_{2}^{*}(t, x) \quad \text { on }(0, T) \times \partial \Omega_{1} .
$$

According to (2.21), combining (2.33), (2.34) and (2.35), we obtain that $\left(z_{1}^{*}, z_{2}^{*}\right)$ coincide with the solution $\left(Z, z_{2}\right)$ of $-\left(Z, z_{2}\right)^{\prime}+\tilde{A}_{*, \sigma_{2}}\left(Z, z_{2}\right)=\left(G, g_{2}\right)$ in $(0, T)$ and $\left(Z, z_{2}\right)(T)=0$. Since this solution is unique, $\left(Z, z_{2}\right)$ is the unique possible weak limit of the sequence $\left(z_{1, \sigma}, z_{2, \sigma}\right)_{\sigma_{1} \geqslant 1}$ as $\sigma_{1} \rightarrow \infty$, hence the whole sequence is weakly convergent in $L^{2}\left(0, T ; H^{1}\left(\Omega_{1}\right)\right) \cap H^{1}\left(0, T ; L^{2}\left(\Omega_{1}\right)\right) \times L^{2}\left(0, T ; H^{2}\left(\Omega_{2}\right)\right) \cap H^{1}\left(0, T ; L^{2}\left(\Omega_{2}\right)\right)$. Thus, by Rellich's compactness embedding theorem

$$
\left(z_{1, \sigma}, z_{2, \sigma}\right) \underset{\sigma_{1} \rightarrow \infty}{\longrightarrow}\left(Z, z_{2}\right) \text { strongly in } L^{2}\left(0, T ; L^{2}\left(\Omega_{1}\right)\right) \times L^{2}\left(0, T ; L^{2}\left(\Omega_{2}\right)\right) .
$$

Similarly, the trace operator from $H^{1}((0, T) \times \Omega)$ to $L^{2}(\{0\} \times \Omega)$ being compact,

$$
\left(z_{1, \sigma}(0, \cdot), z_{2, \sigma}(0, \cdot)\right) \underset{\sigma_{1} \rightarrow \infty}{\longrightarrow}\left(Z(0), z_{2}(0, \cdot)\right) \text { strongly in } L^{2}\left(\Omega_{1}\right) \times L^{2}\left(\Omega_{2}\right) .
$$

These convergences finish the proof of Lemma 2.3.

\subsection{Well-posedness issues for wave equation with discontinuous velocities}

Here, we recall some facts on the well-posedness of the wave equation

$$
\begin{cases}\partial_{t}^{2} w_{\sigma}-\operatorname{div}\left(\sigma \nabla w_{\sigma}\right)=0, & \text { in }(0, T) \times \Omega, \\ w_{\sigma}=0, & \text { on }(0, T) \times \partial \Omega \\ \left(w_{\sigma}, \partial_{t} w_{\sigma}\right)(0)=\left(w^{0}, w^{1}\right), & \text { in } \Omega,\end{cases}
$$

for a general $\sigma \in L^{\infty}(\Omega)$ satisfying $(2.2)$ and initial data $\left(w^{0}, w^{1}\right)$ in $H_{0}^{1}(\Omega) \times L^{2}(\Omega)$.

Note that, following the notation used in Section 2.1, (2.36) can be reformulated as

$$
w_{\sigma}^{\prime \prime}+\tilde{A}_{\sigma} w_{\sigma}=0 \text { in }(0, T), \quad\left(w_{\sigma}(0), w_{\sigma}^{\prime}(0)\right)=\left(w^{0}, w^{1}\right)
$$

with $\tilde{A}_{\sigma}$ defined in $(2.6)-(2.7)$. As $\tilde{A}_{\sigma}$ is a positive self-adjoint operator, for $\left(w_{0}, w_{1}\right) \in \mathcal{D}\left(\tilde{A}_{\sigma}^{1 / 2}\right) \times L^{2}(\Omega)$, there exists a unique solution $w_{\sigma}$ of (2.37) such that

$$
w_{\sigma} \in C^{0}\left([0, T] ; \mathcal{D}\left(\tilde{A}_{\sigma}^{1 / 2}\right)\right) \cap C^{1}\left([0, T] ; L^{2}(\Omega)\right)=C^{0}\left([0, T] ; H_{0}^{1}(\Omega)\right) \cap C^{1}\left([0, T] ; L^{2}(\Omega)\right) .
$$

Besides, if $\left(w_{0}, w_{1}\right) \in \mathcal{D}\left(\tilde{A}_{\sigma}\right) \times \mathcal{D}\left(\tilde{A}_{\sigma}^{1 / 2}\right)$, the solution $w_{\sigma}$ of $(2.37)$ satisfies

$$
w_{\sigma} \in C^{0}\left([0, T] ; \mathcal{D}\left(\tilde{A}_{\sigma}\right)\right) \cap C^{1}\left([0, T] ; \mathcal{D}\left(\tilde{A}_{\sigma}^{1 / 2}\right)\right) \cap C^{2}\left([0, T] ; L^{2}(\Omega)\right) .
$$

Since we want to keep track of the dependences of the solutions with respect to the coefficient $\sigma$, we need to be slightly more precise than that in the following study. 
Namely, we introduce the energy of solutions of (2.36) given for $t>0$ by

$$
E_{\sigma}(t)=\frac{1}{2} \int_{\Omega} \sigma\left|\nabla w_{\sigma}(t)\right|^{2} \mathrm{~d} x+\frac{1}{2} \int_{\Omega}\left|\partial_{t} w_{\sigma}(t)\right|^{2} \mathrm{~d} x
$$

One classicaly checks that it is a preserved quantity, or in other words that for all $\left(w_{0}, w_{1}\right) \in H_{0}^{1}(\Omega) \times L^{2}(\Omega)$, and all $t \in[0, T]$,

$$
E_{\sigma}(t)=E_{\sigma}(0)
$$

To conclude this section, let us also mention the following hidden regularity property (see [24], p. 371): when $\sigma$ is $C^{1}$ in a neighborhood $\Gamma$ of a part of $\partial \Omega$, the solutions $w_{\sigma}$ of $(2.36)$ with initial data $\left(w_{0}, w_{1}\right) \in H_{0}^{1}(\Omega) \times L^{2}(\Omega)$ satisfy $\sigma \partial_{\nu} w_{\sigma} \in L^{2}\left(0, T ; L^{2}(\Gamma)\right)$.

\section{Proofs of Theorem 1.1 And Corollary 1.3}

In this section, we prove the main result of this article, i.e. Theorem 1.1 and its corollary, Corollary 1.3. Roughly speaking, the plan of the proof of Theorem 1.1 can be described as follows:

- We focus on the proof of the uniform observability inequality of the wave equation with respect to $\sigma_{1}$, i.e., we prove Theorem 1.4. This will be done using multiplier techniques.

- We apply the transmutation method as in [11] to prove a uniform observability inequality for the heat equation where the observation is given in a part of the boundary.

- Using an auxiliary cut-off function and a Cacciopoli inequality, we replace the norm of the boundary by the $L^{2}\left(0, T ; L^{2}(\omega)\right)$ norm in the previously obtained observability inequality.

For simplicity, the proof is divided into several steps that will be presented in different paragraphs. The proof of Corollary 1.3 is then presented in the last paragraph of the section.

Notations and convention. In the following, the coefficient $\sigma_{2}$ is assumed to be fixed to a positive constant. The coefficient $\sigma_{1}$ however is simply larger than $\sigma_{2}$, and the constants are all independent of $\sigma_{1}$.

\subsection{Proof of Theorem 1.4: Uniform observability of the wave equation}

In this step, we study uniform observability properties for the wave equation (1.13), that is to say, we will prove the existence of a constant $C=C\left(\Omega, \Gamma_{0}, T, \sigma_{2}\right)$ independent of $\sigma_{1}\left(\geqslant \sigma_{2}\right)$ such that

$$
E_{\sigma}(0) \leqslant C \int_{0}^{T} \int_{\Gamma_{0}}\left|\partial_{\nu} w_{\sigma}\right|^{2} \mathrm{~d} \gamma \mathrm{d} t
$$

for all $w_{\sigma}$ solution of (1.13) and $E_{\sigma}$ defined in (2.38). As said in the introduction, our proof follows the one in Chapter 6 of [24], keeping track of the dependence of the constants in terms of $\sigma_{1}$.

By a usual density argument, it is sufficient to consider the case of initial conditions lying in $\mathcal{D}\left(\tilde{A}_{\sigma}\right) \times$ $\mathcal{D}\left(\tilde{A}_{\sigma}^{1 / 2}\right)$, for which the solution $w_{\sigma}$ of $(1.13)$ belongs to the space $C^{0}\left([0, T] ; \mathcal{D}\left(\tilde{A}_{\sigma}\right)\right) \cap C^{1}\left([0, T] ; \mathcal{D}\left(\tilde{A}_{\sigma}^{1 / 2}\right)\right) \cap$ $C^{2}\left([0, T] ; L^{2}(\Omega)\right)$.

In order to simplify our notation, in this step we will ignore the dependence of $w_{\sigma}$ in $\sigma$, so we simply write $w$ instead of $w_{\sigma}$, but all the constants appearing next will be independent of $\sigma_{1}$. In addition, we suppose without loss of generality that $x_{0}=\mathbf{0}_{\mathbb{R}^{d}}$. 
Since for $w \in \mathcal{D}\left(\tilde{A}_{\sigma}\right)$, Lemma 2.1 implies that we have $\left(w_{1}, w_{2}\right)=\left(\left.w\right|_{\Omega_{1}},\left.w\right|_{\Omega_{2}}\right) \in H^{2}\left(\Omega_{1}\right) \times H^{2}\left(\Omega_{2}\right)$, it is natural to consider the equation satisfied by $w_{1}$ in $(0, T) \times \Omega_{1}$ and by $w_{2}$ in $(0, T) \times \Omega_{2}$ :

$$
\begin{array}{ll}
\partial_{t}^{2} w_{1}-\sigma_{1} \Delta w_{1}=0 & \text { in }(0, T) \times \Omega_{1}, \\
\partial_{t}^{2} w_{2}-\sigma_{2} \Delta w_{2}=0 & \text { in }(0, T) \times \Omega_{2} .
\end{array}
$$

There, in view of the aforementioned regularity, we can develop the usual multiplier argument, which consists in multiplying the equation (3.2) by $x \cdot \nabla w_{1}+(d-1) w_{1} / 2$ and the equation (3.3) by $x \cdot \nabla w_{2}+(d-1) w_{2} / 2$ and do integration by parts.

These classical computations yield, see e.g. [17, 24], for $i \in\{1,2\}$,

$$
\begin{aligned}
& \left.\int_{\Omega_{i}} \partial_{t} w_{i}(t)\left(x \cdot \nabla w_{i}(t)+\frac{d-1}{2} w_{i}\right)\right|_{0} ^{T} \mathrm{~d} x+\frac{1}{2} \int_{0}^{T} \int_{\Omega_{i}}\left(\left|\partial_{t} w_{i}\right|^{2}+\sigma_{i}\left|\nabla w_{i}\right|^{2}\right) \mathrm{d} x \mathrm{~d} t \\
& +\frac{1}{2} \int_{0}^{T} \int_{\partial \Omega_{i}} x \cdot \nu_{i}\left(\sigma_{i}\left|\nabla w_{i}\right|^{2}-\left|\partial_{t} w_{i}\right|^{2}\right) \mathrm{d} \gamma \mathrm{d} t-\int_{0}^{T} \int_{\partial \Omega_{i}} \sigma_{i} \partial_{\nu_{i}} w_{i}\left(x \cdot \nabla w_{i}+\frac{d-1}{2} w_{i}\right) \mathrm{d} \gamma \mathrm{d} t=0
\end{aligned}
$$

where $\nu_{i}$ denotes the outward normal to $\Omega_{i}$. Recalling that we chose $\nu=\nu_{2}=-\nu_{1}$ on $\partial \Omega_{1} \cap \partial \Omega_{2}$, summing up the two above identities and using the boundary conditions, we get

$$
\begin{aligned}
& \left.\sum_{i=1}^{2} \int_{\Omega_{i}} \partial_{t} w_{i}(t)\left(x \cdot \nabla w_{i}(t)+\frac{d-1}{2} w_{i}\right)\right|_{0} ^{T} \mathrm{~d} x+\frac{1}{2} \sum_{i=1}^{2} \int_{0}^{T} \int_{\Omega_{i}}\left(\left|\partial_{t} w_{i}\right|^{2}+\sigma_{i}\left|\nabla w_{i}\right|^{2}\right) \mathrm{d} x \mathrm{~d} t \\
& \quad+\frac{1}{2} \int_{0}^{T} \int_{\partial \Omega_{1}} x \cdot \nu\left(\left(\sigma_{2}\left|\nabla w_{2}\right|^{2}-\left|\partial_{t} w_{2}\right|^{2}\right)-\left(\sigma_{1}\left|\nabla w_{1}\right|^{2}-\left|\partial_{t} w_{1}\right|^{2}\right)\right) \mathrm{d} \gamma \mathrm{d} t \\
& \quad+\int_{0}^{T} \int_{\partial \Omega_{1}}\left(\sigma_{1} \partial_{\nu} w_{1}\left(x \cdot \nabla w_{1}+\frac{d-1}{2} w_{1}\right)-\sigma_{2} \partial_{\nu} w_{2}\left(x \cdot \nabla w_{2}+\frac{d-1}{2} w_{2}\right)\right) \mathrm{d} \gamma \mathrm{d} t \\
& =\frac{1}{2} \int_{0}^{T} \int_{\partial \Omega} x \cdot \nu \sigma_{2}\left|\partial_{\nu} w_{2}\right|^{2} \mathrm{~d} \gamma \mathrm{d} t,
\end{aligned}
$$

where we have used that $w_{2}=0$ on $(0, T) \times \partial \Omega$.

We now focus on the integrals over $(0, T) \times \partial \Omega_{1}$. There, using the interface conditions

$$
w_{1}=w_{2} \text { and } \sigma_{1} \partial_{\nu} w_{1}=\sigma_{2} \partial_{\nu} w_{2} \quad \text { on }(0, T) \times \partial \Omega_{1}
$$

we can write:

$$
\begin{aligned}
\frac{1}{2} \int_{0}^{T} \int_{\partial \Omega_{1}} x & \cdot \nu\left(\left(\sigma_{2}\left|\nabla w_{2}\right|^{2}-\left|\partial_{t} w_{2}\right|^{2}\right)-\left(\sigma_{1}\left|\nabla w_{1}\right|^{2}-\left|\partial_{t} w_{1}\right|^{2}\right)\right) \mathrm{d} \gamma \mathrm{d} t \\
& +\int_{0}^{T} \int_{\partial \Omega_{1}}\left(\sigma_{1} \partial_{\nu} w_{1}\left(x \cdot \nabla w_{1}+\frac{d-1}{2} w_{1}\right)-\sigma_{2} \partial_{\nu} w_{2}\left(x \cdot \nabla w_{2}+\frac{d-1}{2} w_{2}\right)\right) \mathrm{d} \gamma \mathrm{d} t \\
& =\frac{1}{2} \int_{0}^{T} \int_{\partial \Omega_{1}} x \cdot \nu\left(\sigma_{2}\left|\nabla w_{2}\right|^{2}-\sigma_{1}\left|\nabla w_{1}\right|^{2}\right) \mathrm{d} \gamma \mathrm{d} t+\int_{0}^{T} \int_{\partial \Omega_{1}}\left(\sigma_{2} \partial_{\nu} w_{2}\left(x \cdot\left(\nabla w_{1}-\nabla w_{2}\right)\right)\right) \mathrm{d} \gamma \mathrm{d} t .
\end{aligned}
$$

Next, we go further and recall that the interface condition $w_{1}=w_{2}$ implies that

$$
\nabla w_{1}=\nu \partial_{\nu} w_{1}+\tau, \quad \nabla w_{2}=\nu \partial_{\nu} w_{2}+\tau, \quad \text { on }(0, T) \times \partial \Omega_{1},
$$


where $\tau$ is a vector field tangential to $\partial \Omega_{1}$. In particular, with the identity $\sigma_{1} \partial_{\nu} w_{1}=\sigma_{2} \partial_{\nu} w_{2}$ on $(0, T) \times \partial \Omega_{1}$, we get

$$
\begin{aligned}
& \left|\nabla w_{1}\right|^{2}=\left|\partial_{\nu} w_{1}\right|^{2}+|\tau|^{2}=\left(\frac{\sigma_{2}}{\sigma_{1}}\right)^{2}\left|\partial_{\nu} w_{2}\right|^{2}+|\tau|^{2} \quad \text { on }(0, T) \times \partial \Omega_{1}, \\
& \left|\nabla w_{2}\right|^{2}=\left|\partial_{\nu} w_{2}\right|^{2}+|\tau|^{2} \quad \text { on }(0, T) \times \partial \Omega_{1}, \\
& x \cdot \nu\left(\sigma_{2}\left|\nabla w_{2}\right|^{2}-\sigma_{1}\left|\nabla w_{1}\right|^{2}\right)=\left(\partial_{\nu} w_{2}\right)^{2} x \cdot \nu\left(\sigma_{2}-\frac{\sigma_{2}^{2}}{\sigma_{1}}\right)+|\tau|^{2} x \cdot \nu\left(\sigma_{2}-\sigma_{1}\right), \quad \text { on }(0, T) \times \partial \Omega_{1}, \\
& \sigma_{2} \partial_{\nu} w_{2}\left(x \cdot\left(\nabla w_{1}-\nabla w_{2}\right)\right)=\left(\partial_{\nu} w_{2}\right)^{2} x \cdot \nu\left(\frac{\sigma_{2}^{2}}{\sigma_{1}}-\sigma_{2}\right) \quad \text { on }(0, T) \times \partial \Omega_{1},
\end{aligned}
$$

so that

$$
\begin{aligned}
\frac{1}{2} \int_{0}^{T} \int_{\partial \Omega_{1}} x \cdot \nu\left(\sigma_{2}\left|\nabla w_{2}\right|^{2}-\sigma_{1}\left|\nabla w_{1}\right|^{2}\right) \mathrm{d} \gamma \mathrm{d} t+\int_{0}^{T} \int_{\partial \Omega_{1}}\left(\sigma_{2} \partial_{\nu} w_{2}\left(x \cdot\left(\nabla w_{1}-\nabla w_{2}\right)\right)\right) \mathrm{d} \gamma \mathrm{d} t & \\
& \geqslant \int_{0}^{T} \int_{\partial \Omega_{1}} \frac{1}{2} x \cdot \nu\left(\sigma_{2}-\sigma_{1}\right)\left(|\tau|^{2}+\left|\partial_{\nu} w_{2}\right|^{2} \frac{\sigma_{2}}{\sigma_{1}}\right) \mathrm{d} \gamma \mathrm{d} t \geqslant 0
\end{aligned}
$$

where the last inequality holds since

- $\Omega_{1}$ is star shaped with respect to 0 , i.e. $x \cdot \nu_{1}=-x \cdot \nu \geqslant 0$ for all $x \in \partial \Omega_{1}$,

$-\sigma_{1} \geqslant \sigma_{2}$.

Estimate (3.5) then yields

$$
\begin{aligned}
\int_{\Omega} \partial_{t} w(t)(x \cdot \nabla w(t) & \left.+\frac{d-1}{2} w\right)\left.\right|_{0} ^{T} \mathrm{~d} x+\frac{1}{2} \int_{0}^{T} \int_{\Omega}\left(\left|\partial_{t} w\right|^{2}+\sigma|\nabla w|^{2}\right) \mathrm{d} x \mathrm{~d} t \\
= & \left.\int_{\Omega} \partial_{t} w(t)\left(x \cdot \nabla w(t)+\frac{d-1}{2} w\right)\right|_{0} ^{T} \mathrm{~d} x+T E_{\sigma}(0) \leqslant \frac{\sigma_{2}}{2} \int_{0}^{T} \int_{\Gamma_{0}} x \cdot \nu\left|\partial_{\nu} w_{2}\right|^{2} \mathrm{~d} \gamma \mathrm{d} t
\end{aligned}
$$

where we have used that the energy of the wave equation $E_{\sigma}$ defined in $(2.38)$ is independent of $t \in[0, T]$.

In order to conclude, let us recall that for $w \in H_{0}^{1}(\Omega)$,

$$
\begin{aligned}
\int_{\Omega}\left|x \cdot \nabla w+\frac{d-1}{2} w\right|^{2} \mathrm{~d} x & =\int_{\Omega}\left(|x \cdot \nabla w|^{2}+\left(\frac{d-1}{2}\right)^{2}|w|^{2}+(d-1) x \cdot \nabla w w\right) \mathrm{d} x \\
& =\int_{\Omega}\left(|x \cdot \nabla w|^{2}+\left(\frac{d-1}{2}\right)^{2}|w|^{2}-\frac{d(d-1)}{2}|w|^{2}\right) \mathrm{d} x \\
& \leqslant \int_{\Omega}|x|^{2}|\nabla w|^{2} \mathrm{~d} x \leqslant \frac{\sup _{\Omega}|x|^{2}}{\sigma_{2}} \int_{\Omega} \sigma|\nabla w|^{2} \mathrm{~d} x .
\end{aligned}
$$

Therefore, for all $t \in[0, T]$,

$$
\begin{aligned}
\left|\int_{\Omega} \partial_{t} w(t)\left(x \cdot \nabla w(t)+\frac{d-1}{2} w\right)\right| \mathrm{d} x & \leqslant\left(\frac{\sup _{\Omega}|x|}{\sqrt{\sigma_{2}}}\right)\left(\frac{1}{2} \int_{\Omega}\left|\partial_{t} w(t)\right|^{2} \mathrm{~d} x+\frac{1}{2} \int_{\Omega} \sigma|\nabla w(t)|^{2} \mathrm{~d} x\right) \\
& \leqslant\left(\frac{\sup _{\Omega}|x|}{\sqrt{\sigma_{2}}}\right) E_{\sigma}(0) .
\end{aligned}
$$


The estimate (3.6) then provides:

$$
2\left(T-2 \frac{\sup _{\Omega}|x|}{\sqrt{\sigma_{2}}}\right) E_{\sigma}(0) \leqslant \sigma_{2} \int_{0}^{T} \int_{\Gamma_{0}} x \cdot \nu\left|\partial_{\nu} w_{2}\right|^{2} \mathrm{~d} \gamma \mathrm{d} t .
$$

This concludes the proof of Theorem 1.4.

\subsection{Proof of Theorem 1.1, Step 2: From heat processes to waves}

Our goal here is to prove the following result:

Proposition 3.1. Let $\sigma_{2}>0$ and assume Assumptions (A1) and (A2).

For all $T>0$, there exists a constant $C=C\left(\Omega, \Gamma_{0}, T, \sigma_{2}\right)>0$ such that for all $\sigma_{1} \geqslant \sigma_{2}$, the solution $z_{\sigma}$ of (1.11) with $z_{0} \in H_{0}^{1}(\Omega)$ satisfies

$$
\left\|z_{\sigma}(T)\right\|_{L^{2}(\Omega)} \leqslant C\left\|\partial_{\nu} z_{\sigma}\right\|_{L^{2}\left((0, T) \times \Gamma_{0}\right)} .
$$

Proof. The argument here is based on Section 3.1 of [11]. As explained there, according to Proposition 3.2 of [11], setting

$$
S \in\left(\frac{\sup _{\Omega}|x|}{\sqrt{\sigma_{2}}}, \frac{2}{\sqrt{3}} \frac{\sup _{\Omega}|x|}{\sqrt{\sigma_{2}}}\right)
$$

we can construct a kernel $\mathcal{K}=\mathcal{K}(t, s)$ solution of

$$
\begin{cases}\partial_{t} \mathcal{K}+\partial_{s}^{2} \mathcal{K}=0, & \text { for }(t, s) \in(0, T) \times(-S, S) \\ \mathcal{K}(0, s)=\mathcal{K}(T, s)=0, & \text { for } s \in(-S, S)\end{cases}
$$

and such that

$$
\mathcal{K}(t, 0)=0, \quad \partial_{s} \mathcal{K}(t, 0)=\exp \left(-\alpha\left(\frac{1}{t}+\frac{1}{T-t}\right)\right), \quad \text { in }(0, T),
$$

where

$$
\alpha=4 \frac{\sup _{\Omega}|x|^{2}}{\sigma_{2}}
$$

and for which we have the estimate:

$$
|\mathcal{K}(t, s)| \leqslant|s| \exp \left(\frac{1}{\min \{t, T-t\}}\left(2 s^{2}-\frac{2 \alpha}{3}\right)\right), \quad(t, s) \in(0, T) \times(-S, S) .
$$

Now, let $z_{0} \in H_{0}^{1}(\Omega)$ and let $z_{\sigma}$ be the solution of (1.11). Then set

$$
w_{\sigma}(s, x)=\int_{0}^{T} \mathcal{K}(t, s) z_{\sigma}(t, x) \mathrm{d} x, \quad s \in(-S, S), x \in \Omega
$$


Easy computations show that $w_{\sigma}$ satisfies the wave equation

$$
\begin{cases}\partial_{s}^{2} w_{\sigma}-\operatorname{div}\left(\sigma \nabla w_{\sigma}\right)=0, & \text { in }(-S, S) \times \Omega \\ w_{\sigma}=0, & \text { on }(-S, S) \times \partial \Omega \\ \left(w_{\sigma}, \partial_{s} w_{\sigma}\right)(0)=\left(0, w_{\sigma}^{1}\right), & \text { in } \Omega\end{cases}
$$

with

$$
w_{\sigma}^{1}=\int_{0}^{T} \exp \left(-\alpha\left(\frac{1}{t}+\frac{1}{T-t}\right)\right) z_{\sigma}(t) \mathrm{d} t
$$

and

$$
\partial_{\nu} w_{\sigma}(s, x)=\int_{0}^{T} \mathcal{K}(t, s) \partial_{\nu} z_{\sigma}(t, x) \mathrm{d} t, \quad \text { in }(-S, S) \times \Gamma_{0}
$$

Applying the uniform observability obtained in Theorem 1.4 to $W_{\sigma}(\tau)=w_{\sigma}(\tau-S)$ for $\tau \in(0,2 S)$, and using that the energy is constant, easily leads to the existence of a constant $C$ independent of $\sigma_{1} \geqslant \sigma_{2}$ such that

$$
\left\|\int_{0}^{T} \exp \left(-\alpha\left(\frac{1}{t}+\frac{1}{T-t}\right)\right) z_{\sigma}(t) \mathrm{d} t\right\|_{L^{2}(\Omega)}^{2} \leqslant C \int_{-S}^{S} \int_{\Gamma_{0}}\left|\int_{0}^{T} \mathcal{K}(t, s) \partial_{\nu} z_{\sigma}(t, x) \mathrm{d} t\right|^{2} \mathrm{~d} \gamma \mathrm{d} s .
$$

On one hand, as, for all $\mu \geqslant 0$, one has

$$
\begin{aligned}
\int_{0}^{T} \exp \left(-\alpha\left(\frac{1}{t}+\frac{1}{T-t}\right)\right) \exp (-\mu t) \mathrm{d} t & \geqslant \exp (-\mu T) \int_{\frac{T}{3}}^{\frac{2 T}{3}} \exp \left(-\alpha\left(\frac{1}{t}+\frac{1}{T-t}\right)\right) \mathrm{d} t \\
& \geqslant \exp (-\mu T) \frac{T}{3} \exp \left(-\frac{9 \alpha}{2 T}\right)
\end{aligned}
$$

decomposing $z_{\sigma}(t)$ on the orthonormal basis formed by the eigenfunctions of the positive self-adjoint operator $\tilde{A}_{\sigma}$ easily leads to

$$
\frac{T^{2}}{9} e^{-9 \alpha / T}\left\|z_{\sigma}(T)\right\|_{L^{2}(\Omega)}^{2} \leqslant\left\|\int_{0}^{T} \exp \left(-\alpha\left(\frac{1}{t}+\frac{1}{T-t}\right)\right) z_{\sigma}(t) \mathrm{d} t\right\|_{L^{2}(\Omega)}^{2} .
$$

On the other hand, the right-hand side of (3.16) can be easily bounded as well since $\mathcal{K}$ is uniformly bounded by $S$ in $(-S, S) \times(0, T)($ recall $(3.11))$ :

$$
\int_{-S}^{S} \int_{\Gamma_{0}}\left|\int_{0}^{T} \mathcal{K}(t, s) \partial_{\nu} z_{\sigma}(t, x) \mathrm{d} t\right|^{2} \mathrm{~d} \gamma \mathrm{d} s \leqslant 2 S^{3} \int_{0}^{T} \int_{\Gamma_{0}}\left|\partial_{\nu} z_{\sigma}(t, x)\right|^{2} \mathrm{~d} \gamma \mathrm{d} t
$$

Combining the last estimates, we easily deduce the observability inequality (3.8).

\subsection{Proof of Theorem 1.1, Step 3: A cut-off argument}

Our goal now is to prove the following result: 
Proposition 3.2. Let $\sigma_{2}>0$ and assume Assumptions (A1) and (A2).

For all $T>0$, there exists a constant $C=C\left(\Omega, \omega, T, \sigma_{2}\right)>0$ such that for all $\sigma_{1} \geqslant \sigma_{2}$, the solution $z_{\sigma}$ of (1.11) with $z_{0} \in H_{0}^{1}(\Omega)$ satisfies (1.12).

Proof. We first claim that Proposition 3.1 and a simple superposition argument implies that there exists a constant $C$ such that for any $\sigma_{1} \geqslant \sigma_{2}$, for any $z_{0} \in H_{0}^{1}(\Omega)$ and $f \in L^{2}\left(0, T ; L^{2}(\Omega)\right)$, the solution $z_{\sigma}$ of

$$
\begin{cases}\partial_{t} z_{\sigma}-\operatorname{div}\left(\sigma \nabla z_{\sigma}\right)=f, & \text { in }(0, T) \times \Omega, \\ z_{\sigma}=0, & \text { on }(0, T) \times \partial \Omega, \\ z_{\sigma}(0)=z_{0}, & \text { in } \Omega,\end{cases}
$$

satisfies

$$
\left\|z_{\sigma}(T)\right\|_{L^{2}(\Omega)} \leqslant C\left\|\partial_{\nu} z_{\sigma}\right\|_{L^{2}\left(0, T ; L^{2}\left(\Gamma_{0}\right)\right)}+C\|f\|_{L^{2}\left(0, T ; L^{2}(\Omega)\right)} .
$$

Indeed, to prove this estimate, write $z_{\sigma}=z_{\sigma}^{0}+z_{\sigma}^{f}$, where $z_{\sigma}^{0}$ is the solution of (1.11) and $z_{\sigma}^{f}$ is the solution of

$$
\begin{cases}\partial_{t} z_{\sigma}^{f}-\operatorname{div}\left(\sigma \nabla z_{\sigma}^{f}\right)=f, & \text { in }(0, T) \times \Omega \\ z_{\sigma}^{f}=0, & \text { on }(0, T) \times \partial \Omega \\ z_{\sigma}^{f}(0)=0, & \text { in } \Omega\end{cases}
$$

Using Proposition 3.1, we have

$$
\begin{aligned}
\left\|z_{\sigma}(T)\right\|_{L^{2}(\Omega)} & \leqslant\left\|z_{\sigma}^{0}(T)\right\|_{L^{2}(\Omega)}+\left\|z_{\sigma}^{f}(T)\right\|_{L^{2}(\Omega)} \\
& \leqslant C\left\|\partial_{\nu} z_{\sigma}^{0}\right\|_{L^{2}\left(0, T ; L^{2}\left(\Gamma_{0}\right)\right)}+\left\|z_{\sigma}^{f}(T)\right\|_{L^{2}(\Omega)} \\
& \leqslant C\left\|\partial_{\nu} z_{\sigma}\right\|_{L^{2}\left(0, T ; L^{2}\left(\Gamma_{0}\right)\right)}+C\left\|\partial_{\nu} z_{\sigma}^{f}\right\|_{L^{2}\left(0, T ; L^{2}\left(\Gamma_{0}\right)\right)}+\left\|z_{\sigma}^{f}(T)\right\|_{L^{2}(\Omega)},
\end{aligned}
$$

where $C$ is the uniform constant given in Proposition 3.1, and thus does not depend on $\sigma_{1} \geqslant \sigma_{2}$. Then we use the energy estimates (2.9)-(2.10)-(2.11) to deduce that there exists a constant $C$ independent of $\sigma_{1} \geqslant \sigma_{2}$ such that

$$
\left\|z_{\sigma}^{f}(T)\right\|_{L^{2}(\Omega)}+\left\|z_{\sigma}^{f}\right\|_{L^{2}\left(0, T ; H^{1}\left(\Omega_{2}\right)\right)}+\left\|\Delta z_{\sigma}^{f}\right\|_{L^{2}\left(0, T ; L^{2}\left(\Omega_{2}\right)\right)} \leqslant C\|f\|_{L^{2}\left(0, T ; L^{2}(\Omega)\right)} .
$$

Therefore we easily deduce that $z_{\sigma}^{f}$ can be estimated in $L^{2}\left(0, T ; H^{2}\left(\mathcal{O}_{2}\right)\right)$ for any neighborhood $\mathcal{O}_{2}$ of $\Gamma_{0}$ included in $\Omega_{2}$ and its normal derivative on $(0, T) \times \Gamma_{0}$ can be estimated as follows:

$$
\left\|\partial_{\nu} z_{\sigma}^{f}\right\|_{L^{2}\left(0, T ; L^{2}\left(\Gamma_{0}\right)\right)} \leqslant C\|f\|_{L^{2}\left(0, T ; L^{2}(\Omega)\right)},
$$

for some constant independent of $\sigma_{1}$. Using then (3.20), we easily conclude the proof of (3.18).

The second step is to prove that taking

$$
\omega_{0}=\left\{x \in \Omega, d\left(x, \Gamma_{0}\right) \leqslant \varepsilon / 2\right\},
$$

for all $T>0$, there exists a constant $C$ such that for any $\sigma_{1} \geqslant \sigma_{2}$, for any $z_{0} \in H_{0}^{1}(\Omega)$, the solution $z_{\sigma}$ of $(1.11)$ satisfies

$$
\left\|z_{\sigma}(T)\right\|_{L^{2}(\Omega)} \leqslant C\left\|z_{\sigma}\right\|_{L^{2}\left(0, T ; H^{1}\left(\omega_{0}\right)\right)}
$$


In order to do that, we choose a cut-off function $\eta \in \mathscr{C}^{\infty}(\bar{\Omega})$, equal to 1 in $\overline{\Omega \backslash \omega_{0}}$ and vanishing close to $\Gamma_{0}$. Applying then (3.18) to $\eta z_{\sigma}$, we get

$$
\left\|\eta z_{\sigma}(T)\right\|_{L^{2}(\Omega)} \leqslant C\left\|z_{\sigma}\right\|_{L^{2}\left(0, T ; H^{1}\left(\omega_{0}\right)\right)} .
$$

The function $(1-\eta) z_{\sigma}$ then satisfies the usual heat equation with Dirichlet boundary condition in $(0, T) \times \Omega_{2}$, with a source term bounded in $L^{2}\left(0, T ; L^{2}\left(\Omega_{2}\right)\right)$ by $\left\|z_{\sigma}\right\|_{L^{2}\left(0, T ; H^{1}\left(\omega_{0}\right)\right)}$. Therefore, the usual observability estimate for the heat equation yields

$$
\left\|(1-\eta) z_{\sigma}(T)\right\|_{L^{2}\left(\Omega_{2}\right)} \leqslant C\left\|z_{\sigma}\right\|_{L^{2}\left(0, T ; H^{1}\left(\omega_{0}\right)\right)},
$$

where the constant $C$ does not depend on $\sigma_{1}$.

Estimate (3.21) then follows immediately from (3.22) and (3.23).

The last step is to use Cacciopoli inequality to replace the norm $L^{2}\left(0, T ; H^{1}\left(\omega_{0}\right)\right)$ in the right hand-side of (3.21) by the $L^{2}\left(0, T ; L^{2}(\omega)\right)$ norm.

In fact, we start by using the observability inequality (3.21) between the times $T / 3$ and $2 T / 3$ : we obtain, for some $C$ independent of $\sigma_{1} \geqslant \sigma_{2}$ that

$$
\left\|z_{\sigma}(2 T / 3)\right\|_{L^{2}(\Omega)} \leqslant C\left\|z_{\sigma}\right\|_{L^{2}\left(T / 3,2 T / 3 ; H^{1}\left(\omega_{0}\right)\right)} .
$$

Since the $L^{2}(\Omega)$ norm of $z_{\sigma}(t, \cdot)$ solution of $(1.11)$ is decreasing, we deduce that

$$
\left\|z_{\sigma}(T)\right\|_{L^{2}(\Omega)} \leqslant C\left\|z_{\sigma}\right\|_{L^{2}\left(T / 3,2 T / 3 ; H^{1}\left(\omega_{0}\right)\right)} .
$$

We then choose a function $\rho \in \mathscr{C}^{\infty}([0, T] \times \bar{\Omega})$ such that

$$
\begin{cases}\rho(t, x)=0, & \text { for }(t, x) \in[0, T] \times \overline{(\Omega \backslash \omega)}, \\ \rho(t, x)=1, & \text { for }(t, x) \in[T / 3,2 T / 3] \times \overline{\omega_{0}} \\ \rho(0, x)=\rho(T, x)=0, & \text { for } x \in \Omega .\end{cases}
$$

Multiplying the equation (1.11) of $z_{\sigma}$ by $\rho z_{\sigma}$, and using that $\rho \equiv 0$ in $[0, T] \times \overline{\Omega_{1}}$, we easily get by integration by parts that

$$
\int_{0}^{T} \int_{\Omega_{2}} \rho \sigma_{2}\left|\nabla z_{\sigma}\right|^{2} \mathrm{~d} x \mathrm{~d} t \leqslant C \int_{0}^{T} \int_{\Omega_{2}}\left(\left|\partial_{t} \rho\right|+|\Delta \rho| \sigma_{2}\right)\left|z_{\sigma}\right|^{2} \mathrm{~d} x \mathrm{~d} t
$$

Bounding from below the left hand side and from above the right hand side, we easily deduce, with a constant $C$ independent of $\sigma_{1}$, that

$$
\int_{T / 3}^{2 T / 3} \int_{\omega_{0}} \sigma_{2}\left|\nabla z_{\sigma}\right|^{2} \mathrm{~d} x \mathrm{~d} t \leqslant C \int_{0}^{T} \int_{\omega}\left|z_{\sigma}\right|^{2} \mathrm{~d} x \mathrm{~d} t
$$

Combining estimates (3.24) and (3.25), we easily deduce the uniform observability estimates (1.12).

\subsection{Proof of Theorem 1.1: Conclusion}

Theorem 1.1 can now be simply deduced by the duality between the null controllability of (1.3) and the observability inequality (1.12) for the adjoint equation (1.11), and we refer for instance to Theorem 11.2.1 of [31]. 
The uniform observability estimate (1.12) for the adjoint equation (1.11) for $\sigma$ of the form (1.1) with $\sigma_{1} \geqslant \sigma_{2}$, $\sigma_{2}$ being fixed, see Proposition 3.2, thus directly implies Theorem 1.1 by duality.

\subsection{Proof of Corollary 1.3}

Let $\left(Y_{0}, y_{0,2}\right) \in \mathbb{R} \times L^{2}\left(\Omega_{2}\right)$, and introduce $y_{0}=Y_{0} 1_{\Omega_{1}}+y_{0,2} 1_{\Omega_{2}}$. According to Theorem 1.1, for all $\sigma$ as in (1.1) with $\sigma_{1} \geqslant \sigma_{2}, \sigma_{2}$ being fixed, there exists a null-control $u_{\sigma} \in L^{2}\left(0, T ; L^{2}(\omega)\right)$ such that the solution $y_{\sigma}$ of (1.3) satisfies $y_{\sigma}(T)=0$ and the controls $\left(u_{\sigma}\right)_{\sigma_{1} \geqslant \sigma_{2}}$ satisfy the uniform bound (1.7). Therefore, the sequence $\left(u_{\sigma}\right)_{\sigma_{1} \geqslant \sigma_{2}}$ weakly converges up to a subsequence as $\sigma_{1} \rightarrow \infty$ in $L^{2}\left(0, T ; L^{2}(\omega)\right)$ to some $u^{*} \in L^{2}\left(0, T ; L^{2}(\omega)\right)$ satisfying

$$
\left\|u^{*}\right\|_{L^{2}((0, T) \times \omega)} \leqslant C\left\|y_{0}\right\|_{L^{2}(\Omega)} \leqslant C\left\|\left(Y_{0}, y_{0,2}\right)\right\|_{\mathbb{R} \times L^{2}\left(\Omega_{2}\right)} .
$$

We can then pass to the limit in the equations (1.3) satisfied by $y_{\sigma}$, and according to Theorem 2.2 , the sequence $\left(y_{\sigma}\right)_{\sigma_{1} \geqslant \sigma_{2}}$ converges to $y^{*}=Y^{*} 1_{\Omega_{1}}+y_{2}^{*} 1_{\Omega_{2}}$ weakly in $L^{2}\left(0, T ; H_{0}^{1}(\Omega)\right)$, weakly-* in $L^{\infty}\left(0, T ; L^{2}(\Omega)\right)$, and strongly in $C^{0}\left((0, T] ; L^{2}(\Omega)\right)$, where $\left(Y^{*}, y_{2}^{*}\right)$ solves $(2.24)$ with $F_{*}=0$ and $f_{2}^{*}=u^{*} 1_{\omega}$.

According to the above convergences, we also have that $Y^{*}(T)=0$ and $y_{2}^{*}(T)=0$ in $\Omega_{2}$, which means that $u^{*}$ is a null control for the limit equation (1.8) with initial datum $\left(Y_{0}, y_{0,2}\right)$.

\section{FURTHER COMMENTS AND OPEN PROBLEMS}

\subsection{A spectral approach in the $1 \mathrm{~d}$ case}

In the 1-dimensional case, Theorem 1.1 can alternatively be proved using the moment method, i.e. a spectral approach based on suitable properties of the eigenvalues and eigenvectors of the underlying operator, the difficulty being to get uniform estimates with respect to the parameter going to infinity.

To better explain this strategy, for $\varepsilon \in(0,1)$, let us consider the system of equation

$$
\begin{cases}\partial_{t} y_{L}-\partial_{x}^{2} y_{L}=0, & \text { in }(0, T) \times(-1,0), \\ \partial_{t} y_{R}-\frac{1}{\varepsilon^{2}} \partial_{x}^{2} y_{R}=0, & \text { in }(0, T) \times(0,1), \\ y_{L}(t, 0)=y_{R}(t, 0), & \text { on }(0, T), \\ \partial_{x} y_{L}(t, 0)=\frac{1}{\varepsilon^{2}} \partial_{x} y_{R}(t, 0), & \text { on }(0, T), \\ y_{L}(t,-1)=u(t), \quad y_{R}(t, 1)=0, & \text { on }(0, T), \\ \left(y_{L}, y_{R}\right)(0)=\left(y_{0, L}, y_{0, R}\right), & \text { in }(-1,0) \times(0,1),\end{cases}
$$

where the control $u$ is now acting only on the left at $x=-1$.

Note that, strictly speaking the system of equation (4.1) does not correspond to the system considered in (1.3) in $1 \mathrm{~d}$, but it clearly has the same flavor and can be dealt with similarly to the price of some minor additional technical difficulties.

We claim the following counterpart to Theorem 1.1 for system (4.1):

Theorem 4.1. For all $T>0$, there exist $\varepsilon_{0}>0$ and $C>0$ such that for all $\left(y_{0, L}, y_{0, R}\right) \in L^{2}(-1,0) \times L^{2}(0,1)$, for all $\varepsilon \in\left(0, \varepsilon_{0}\right)$, there exists a control $u_{\varepsilon} \in L^{2}(0, T)$, such that the solution $\left(y_{L, \varepsilon}, y_{R, \varepsilon}\right)$ of (4.1) satisfies $\left(y_{L, \varepsilon}(T), y_{R, \varepsilon}(T)\right)=(0,0)$ in $L^{2}(-1,0) \times L^{2}(0,1)$, and

$$
\left\|u_{\varepsilon}\right\|_{L^{2}(0, T)} \leqslant C\left\|\left(y_{0, L}, y_{0, R}\right)\right\|_{L^{2}(-1,0) \times L^{2}(0,1)} .
$$


Remark 4.2. Note that the limit system corresponding to (4.1) in the limit $\varepsilon \rightarrow 0$ simply is

$$
\begin{cases}\partial_{t} y_{L}-\partial_{x}^{2} y_{L}=0, & \text { in }(0, T) \times(-1,0), \\ y_{L}(t, 0)=0, & \text { on }(0, T), \\ y_{L}(t,-1)=u(t), & \text { on }(0, T), \\ y_{L}(0)=y_{0, L}, & \text { in }(-1,0) \times(0,1),\end{cases}
$$

i.e. the heat equation with homogeneous Dirichlet boundary condition at $x=0$, for which null-controllability is well-known (see e.g. [14]).

Also note that homogeneous Neumann boundary conditions $\partial_{x} y_{R}(t, 1)=0$ on $y_{R}$ at $x=1$ in (4.1) would have led in the limit $\varepsilon \rightarrow 0$ to the dynamic boundary condition $\partial_{t} y_{L}(t, 0)+\partial_{x} y_{L}(t, 0)=0$ similarly to (1.8) in $1 \mathrm{~d}$, for which the work ([18], Chap. 5) proves the null-controllability of the corresponding equation.

We will not provide a detailed proof of Theorem 4.1 since it is quite lengthy and could also be done quicker using the observability of the corresponding wave equation and the transmutation technique as we did for the proof of Theorem 1.1.

We will nevertheless briefly sketch it below to explain the key steps to prove Theorem 4.1 using the moment method.

Sketch of the proof of Theorem 4.1. The moment method is a spectral approach, relying on a good knowledge of eigenvalues and eigenvectors. Thus, for all $\varepsilon \in(0,1)$, we define the operator $A_{\varepsilon}$ defined on $L^{2}(-1,0) \times L^{2}(0,1)$ by

$$
A_{\varepsilon}\left(y_{L}, y_{R}\right)=\left(-\partial_{x}^{2} y_{L},-\frac{1}{\varepsilon^{2}} \partial_{x}^{2} y_{R}\right)
$$

with domain

$$
\begin{aligned}
\mathcal{D}\left(A_{\varepsilon}\right)=\left\{\left(y_{L}, y_{R}\right) \in H^{2}(-1,0) \times H^{2}(0,1)\right. \\
\left.\quad \text { with } y_{L}(-1)=y_{R}(1)=0, y_{L}(0)=y_{R}(0), \text { and } \partial_{x} y_{L}(0)=\frac{1}{\varepsilon^{2}} \partial_{x} y_{R}(0)\right\} .
\end{aligned}
$$

Similarly as in Section 2.1, it is classical to check that these operators are self-adjoint, positive definite and with compact resolvent.

Therefore, their spectrum is made by a sequence of strictly positive eigenvalues $\left(\lambda_{k, \varepsilon}\right)_{k \in \mathbb{N}}$ (increasingly ordered) and of corresponding eigenvectors $\left(\Phi_{k, L, \varepsilon}, \Phi_{k, R, \varepsilon}\right)_{k \in \mathbb{N}}$.

Our next goal will be to prove the following statement: There exists $\varepsilon_{0}>0$ small enough such that

- The eigenvalues satisfy:

$$
\inf _{\varepsilon \in\left(0, \varepsilon_{0}\right)} \inf _{k \in \mathbb{N}}\left\{\sqrt{\lambda_{k+1, \varepsilon}}-\sqrt{\lambda_{k, \varepsilon}}\right\}>0 .
$$

- The eigenvectors satisfy:

$$
\inf _{\varepsilon \in\left(0, \varepsilon_{0}\right)} \inf _{k \in \mathbb{N}}\left\{\frac{\left|\partial_{x} \Phi_{k, L, \varepsilon}(-1)\right|}{\sqrt{\lambda_{k, \varepsilon}}\left\|\left(\Phi_{k, L, \varepsilon}, \Phi_{k, R, \varepsilon}\right)\right\|_{L^{2}(-1,0) \times L^{2}(0,1)}}\right\}>0 .
$$


To prove these properties, for $\varepsilon \in(0,1)$, we consider the eigenvalue problem:

$$
\begin{cases}-\partial_{x}^{2} \Phi_{L}=\lambda \Phi_{L} & \text { in }(-1,0), \\ -\frac{1}{\varepsilon^{2}} \partial_{x}^{2} \Phi_{R}=\lambda \Phi_{R} & \text { in }(0,1), \\ \Phi_{L}(0)=\Phi_{R}(0), & \\ \partial_{x} \Phi_{L}(0)=\frac{1}{\varepsilon^{2}} \partial_{x} \Phi_{R}(0), & \\ \Phi_{L}(-1)=\Phi_{R}(1)=0 . & \end{cases}
$$

Setting $\omega=\sqrt{\lambda}$, the first four equations imply, that, for some constants $A$ and $B$,

$$
\begin{aligned}
& \Phi_{L, \varepsilon}(x)=A \cos (\omega x)+B \sin (\omega x), \quad x \in(-1,0), \\
& \Phi_{R, \varepsilon}(x)=A \cos (\varepsilon \omega x)+\varepsilon B \sin (\varepsilon \omega x), \quad x \in(0,1) .
\end{aligned}
$$

The boundary conditions then imply that $A$ and $B$ can be chosen to be non-zero if and only if

$$
\operatorname{Det}\left(\begin{array}{cc}
\cos (\omega) & -\sin (\omega) \\
\cos (\varepsilon \omega) & \varepsilon \sin (\varepsilon \omega)
\end{array}\right)=0
$$

that is if $\omega \geqslant 0$ satisfies

$$
f_{\varepsilon}(\omega)=0 \text { where } f_{\varepsilon}(\rho)=\varepsilon \cos (\rho) \sin (\varepsilon \rho)+\sin (\rho) \cos (\varepsilon \rho), \quad\left(\rho \in \mathbb{R}_{+}^{*}\right) .
$$

In fact, one easily checks that, for all $\varepsilon>0, \lambda>0$ is an eigenvalue of $A_{\varepsilon}$ corresponding to an eigenvector $\left(\Phi_{L}, \Phi_{R}\right)$ if and only if $f_{\varepsilon}(\sqrt{\lambda})=0$, and then the corresponding eigenvector (not normalized) can be given by

$$
\begin{aligned}
& \Phi_{L}(x)=\sin (\sqrt{\lambda}(x+1)), \quad x \in(-1,0), \\
& \Phi_{R}(x)=\frac{\varepsilon \cos (\sqrt{\lambda})}{\cos (\varepsilon \sqrt{\lambda})} \sin (\varepsilon \sqrt{\lambda}(x-1)), \quad x \in(0,1),
\end{aligned}
$$

where $\varepsilon \cos (\sqrt{\lambda}) / \cos (\varepsilon \sqrt{\lambda})$ is replaced by 1 when $\cos (\varepsilon \sqrt{\lambda})=0$ (if $\cos (\varepsilon \sqrt{\lambda})=0$ and $f_{\varepsilon}(\sqrt{\lambda})=0, \cos (\sqrt{\lambda})=0$, and this ratio should thus be seen as the extension by continuity of the map $\rho \mapsto \varepsilon \cos (\rho) / \cos (\varepsilon \rho)$ when the functions $\rho \mapsto \cos (\rho)$ and $\rho \mapsto \cos (\varepsilon \rho)$ cancel in the same point).

Proof of estimate (4.4). In order to prove the estimate (4.4), according to the above discussion, it is sufficient to prove that there exists a constant $\alpha>0$ such that for $\varepsilon>0$ small enough, two positive consecutive root of the function $f_{\varepsilon}$ (defined in (4.7)) are at a distance $\geqslant \alpha$. This is not difficult to check, following the arguments hereafter.

First, remark that if $f_{\varepsilon}(\omega)=0$ and $\cos (\omega)=0$, then $\cos (\varepsilon \omega)=0$, and that if $f_{\varepsilon}(\omega)=0$ and $\cos (\varepsilon \omega)=0$, then $\cos (\omega)=0$. Therefore, it is interesting to write $f_{\varepsilon}$ under the form $f_{\varepsilon}(\rho)=\cos (\rho) \cos (\varepsilon \rho) g_{\varepsilon}(\rho)$ for the function $g_{\varepsilon}$ defined by

$$
g_{\varepsilon}(\rho)=\varepsilon \tan (\varepsilon \rho)+\tan (\rho),
$$

for $\rho \in \mathbb{R}_{+}^{*} \backslash\left(\cup_{k \in \mathbb{N}}\{(k+1 / 2) \pi\} \cup_{\ell \in \mathbb{N}}\{(\ell+1 / 2) \pi / \varepsilon\}\right)$. Accordingly, roots of $f_{\varepsilon}$ are either simultaneous roots of $\cos$ and $\cos (\varepsilon \cdot)$, or roots of $g_{\varepsilon}$.

If we first assume that $\varepsilon$ cannot be written under the form $(2 k+1) /(2 \ell+1)$ for $(k, \ell) \in \mathbb{N}^{2}$, then there are no common roots of $\cos$ and $\cos (\varepsilon \cdot)$, so the equation reduces to the analysis of the roots of $g_{\varepsilon}$. Since $g_{\varepsilon}$ is a 
strictly increasing function apart from the singularities of $\tan$ and $\tan (\varepsilon \cdot)$, it is clear that $g_{\varepsilon}$ has exactly one roots between each of the singularities of $\tan$ and $\tan (\varepsilon \cdot)$.

If $\omega$ is a root of $g_{\varepsilon}$ which belongs to an interval of the form $(-\pi / 2+k \pi, \pi / 2+k \pi)$ with $k \in \mathbb{N}$ such that $\tan (\varepsilon \cdot)$ has no singularity in this interval, then we are in one of the following cases:

- if $\omega<\pi / 4+k \pi$, the next root being after the next singularity of $g_{\varepsilon}$, it necessarily is at a distance at least $\pi / 4$.

- if $\omega>\pi / 4+k \pi$, then $\tan (\omega) \geqslant 1$, and therefore $\varepsilon \tan (\varepsilon \omega) \leqslant-1$. Thus, if $\varepsilon$ is small enough, we necessarily have that $\tan (\varepsilon \cdot)$ is well-defined and negative on $(\pi / 2+k \pi, \pi / 2+(k+1) \pi)$, so that the next root $\omega_{1}$ necessarily satisfies $\omega_{1} \in(\pi / 2+k \pi, \pi / 2+(k+1) \pi)$ and $\tan \left(\omega_{1}\right) \geqslant 0$. Accordingly, $\omega_{1} \geqslant(k+1) \pi$ and $\omega_{1}-\omega \geqslant \pi / 2$.

If $\omega$ is a root of $g_{\varepsilon}$ which belongs to an interval of the form $(-\pi / 2+k \pi, \pi / 2+k \pi)$ with $k \in \mathbb{N}$ such that $\tan (\varepsilon \cdot)$ has a singularity in this interval, of the form $(\ell+1 / 2) \pi / \varepsilon$ with $\ell \in \mathbb{N}$, then

- if $\omega$ is larger than $(\ell+1 / 2) \pi / \varepsilon$; We first check that for $\varepsilon>0$ small enough, $\varepsilon \tan (\varepsilon \rho) \leqslant-1$ for all $\rho \in((\ell+1 / 2) \pi / \varepsilon,(k+3 / 2) \pi)$, so that the next root $\omega_{1}$ of $g_{\varepsilon}$ satisfies $\omega_{1} \in(\pi / 2+k \pi, \pi / 2+(k+1) \pi)$ and $\tan \left(\omega_{1}\right) \geqslant 0$ and thus $\omega_{1} \geqslant(k+1) \pi$ and $\omega_{1}-\omega \geqslant \pi / 2$. Besides, we have that $\tan (\omega) \geqslant 1$.

- if $\omega$ is smaller than $(\ell+1 / 2) \pi / \varepsilon$; We first check that for $\varepsilon>0$ small enough, $\varepsilon \tan (\varepsilon \rho) \geqslant 1$ for all $\rho \in$ $((k-1 / 2) \pi,(\ell+1 / 2) \pi / \varepsilon)$, so that we necessarily have that $\tan (\omega) \leqslant-1$. The previous item shows that the next root $\omega_{1}$ of $g_{\varepsilon}$ belongs to $((\ell+1 / 2) \pi / \varepsilon,(k+1 / 2) \pi)$ and satisfies $\tan \left(\omega_{1}\right) \geqslant 1$. Therefore, $\omega_{1}-\omega$ is necessarily larger than $\pi / 2$.

We have thus proved the uniform spectral gap condition (4.4) when $\varepsilon$ is small enough and not of the form $(2 k+1) /(2 \ell+1)$ for $(k, \ell) \in \mathbb{N}^{2}$. In fact, when $\varepsilon$ is of the form $(2 k+1) /(2 \ell+1)$ for $(k, \ell) \in \mathbb{N}^{2}$, the same arguments as above can be adapted locally around the common roots of $\cos$ and $\cos (\varepsilon \cdot)$ with almost no change, so we leave it to the reader.

Proof of estimate (4.5). According to the formulas (4.8)-(4.9), proving the estimate (4.5) amounts to show that

$$
\sup _{\varepsilon \in\left(0, \varepsilon_{0}\right)} \sup \left\{\left|\frac{\varepsilon \cos (\omega)}{\cos (\varepsilon \omega)}\right|, \text { for } \omega \text { such that } f_{\varepsilon}(\omega)=0\right\}<\infty
$$

Indeed, if (4.11) holds, then the ratio $\left\|\Phi_{k, L, \varepsilon}\right\|_{L^{2}(-1,0)} /\left\|\left(\Phi_{k, L, \varepsilon}, \Phi_{k, R, \varepsilon}\right)\right\|_{L^{2}(-1,0) \times L^{2}(0,1)}$ is uniformly bounded from below for $\varepsilon \in\left(0, \varepsilon_{0}\right)$ and $k \in \mathbb{N}$, and then (4.5) follows from the classical fact that

$$
\inf _{\varepsilon \in\left(0, \varepsilon_{0}\right)} \inf _{k \in \mathbb{N}}\left\{\frac{\left|\partial_{x} \Phi_{k, L, \varepsilon}(-1)\right|}{\sqrt{\lambda_{k, \varepsilon}}|| \Phi_{k, L, \varepsilon} \|_{L^{2}(-1,0)}}\right\}>0 .
$$

which is a straightforward estimate for $\Phi_{k, L, \varepsilon}$ of the form (4.8).

To prove (4.11), we simply remark that $f_{\varepsilon}(\omega)=0$ implies that

$$
\varepsilon^{2} \cos (\omega)^{2} \sin (\varepsilon \omega)^{2}=\sin (\omega)^{2} \cos (\varepsilon \omega)^{2},
$$

hence

$$
\varepsilon^{2} \cos (\omega)^{2}\left(1-\cos (\varepsilon \omega)^{2}\right)=\left(1-\cos (\omega)^{2}\right) \cos (\varepsilon \omega)^{2},
$$


and

$$
\frac{\varepsilon^{2} \cos (\omega)^{2}}{\cos (\varepsilon \omega)^{2}}=\left(1-\left(1-\varepsilon^{2}\right) \cos (\omega)^{2}\right) \leqslant 1
$$

This concludes the proof of property (4.11) and property (4.5).

Conclusion. To conclude the proof of Theorem 4.1, for all $\varepsilon \in\left(0, \varepsilon_{0}\right)$, we can then follow the classical strategy (see e.g. [30] or Chap. IV of [8]), which consists in building biorthogonal families to the exponential functions $\left(t \mapsto \exp \left(-\lambda_{k, \varepsilon} t\right)\right)_{k \in \mathbb{N}}$ and constructing a control $u_{\varepsilon}$ by a suitable combination of these biorthogonal families. The only point to check is the uniform bound on the controls $u_{\varepsilon}$ constructed that way, which will follow from the application of Theorems IV.1.8 and IV.1.9 in [8], whose assumptions are satisfied due the uniform bounds in (4.4) and (4.5). In particular, one easily checks that condition (4.4) implies, by setting

$$
\gamma=\inf _{\varepsilon \in\left(0, \varepsilon_{0}\right)} \inf _{k \in \mathbb{N}}\left\{\sqrt{\lambda_{k+1, \varepsilon}}-\sqrt{\lambda_{k, \varepsilon}}\right\}
$$

that for all $r>0$ and $\varepsilon \in\left(0, \varepsilon_{0}\right)$,

$$
\sum_{\lambda_{k, \varepsilon}>r} \frac{1}{\lambda_{k, \varepsilon}} \leqslant \sum_{k \in \mathbb{N}} \frac{1}{(\sqrt{r}+\gamma k)^{2}}=\frac{1}{r}+\sum_{k=1}^{\infty} \frac{1}{(\sqrt{r}+\gamma k)^{2}} \leqslant \frac{1}{r}+\int_{0}^{\infty} \frac{1}{(\sqrt{r}+\gamma x)^{2}} \mathrm{~d} x \leqslant \frac{1}{r}+\frac{1}{\gamma \sqrt{r}},
$$

so that all families $\left(\lambda_{k, \varepsilon}\right)_{k \in \mathbb{N}}$ have a uniform remainder function. The other conditions of Theorems IV.1.8 and IV.1.9 in [8] follow easily from the condition (4.4) and the fact that the first eigenvalues $\lambda_{1, \varepsilon}$ are uniformly bounded from below.

\subsection{More general geometric conditions}

A very interesting problem is to discuss if the geometric conditions (A1) and (A2) are really needed or not to get uniform controllability results when the conductivity in one of the two medium goes to infinity.

We emphasize that here, these geometric conditions arise because our strategy of proof relies on the controllability of the corresponding wave equation, and are thus probably only technical.

It would be very natural to try to develop suitable Carleman estimates in the spirit of the works [5-7, 20-22] to derive uniform observability estimates (1.12) for the solutions of (1.11) for conductivities $\sigma$ of the form (1.1) when $\sigma_{1} \rightarrow \infty$ under the only condition that the control set $\omega$ is non-empty and $\omega \subset \Omega_{2}$.

This is so far an open problem, even when considering the restrictive geometric setting of [10].

\subsection{The case of a control set in the strongly conductive material}

When considering a control acting in the strongly conductive material, we claim the following result:

Theorem 4.3. Let $\Omega$ be a smooth bounded domain of $\mathbb{R}^{d}, d \geqslant 2, \Omega_{1}$ be a smooth non-empty subdomain of $\Omega$ with $\Omega_{1} \Subset \Omega$, and $\Omega_{2}=\Omega \backslash \overline{\Omega_{1}}, \omega \subset \Omega_{1}, \sigma_{2}>0$, and $T>0$.

Then there is no constant $C$ such that for all $\sigma_{1} \geqslant \sigma_{2}$, any solution $z_{\sigma}$ of (1.11) satisfies the observability inequality

$$
\left\|z_{\sigma}(T)\right\|_{L^{2}(\Omega)} \leqslant C\left\|z_{\sigma}\right\|_{L^{2}((0, T) \times \omega)} .
$$

In particular, the systems (1.3) are not uniformly controllable as $\sigma_{1} \rightarrow \infty$.

Proof. We prove Theorem 4.3 by contradiction, by assuming that the systems (1.11) are uniformly observable and uniformly satisfy the observability inequality (4.12) with $\omega \subset \Omega_{1}$. 
In such case, according to Theorem 2.2 and following the proof of Corollary 1.3, we could deduce the nullcontrollability of the limit system with a scalar control $u^{*}$ acting on the boundary $\partial \Omega_{1}$. To be more precise, this implies that for all $\left(Y_{0}, y_{0,2}\right) \in \mathbb{R} \times L^{2}\left(\Omega_{2}\right)$, there exists a control function $u^{*} \in L^{2}(0, T)$ such that the solution $\left(Y, y_{2}\right)$ of

$$
\begin{cases}\partial_{t} y_{2}-\sigma_{2} \Delta y_{2}=0, & \text { in }(0, T) \times \Omega_{2}, \\ y_{2}(t, x)=0, & \text { on }(0, T) \times \partial \Omega \\ y_{2}(t, x)=Y(t), & \text { on }(0, T) \times \partial \Omega_{1} \\ \partial_{t} Y+\frac{\sigma_{2}}{\left|\Omega_{1}\right|} \int_{\partial \Omega_{1}} \partial_{\nu} y_{2}(t) \mathrm{d} \gamma=u^{*}, & \text { in }(0, T), \\ y_{2}(0, \cdot)=y_{0,2}(\cdot), & \text { in } \Omega_{2}, \\ Y(0)=Y_{0}, & \end{cases}
$$

satisfies $Y(T)=0$ and $y_{2}(T)=0$ in $\Omega_{2}$.

Consequently, this would entail that for all $y_{0,2} \in L^{2}\left(\Omega_{2}\right)$, there exists a control $Y \in L^{2}(0, T)$ such that the solution $y_{2}$ of

$$
\begin{cases}\partial_{t} y_{2}-\sigma_{2} \Delta y_{2}=0, & \text { in }(0, T) \times \Omega_{2}, \\ y_{2}(t, x)=0, & \text { on }(0, T) \times \partial \Omega, \\ y_{2}(t, x)=Y(t), & \text { on }(0, T) \times \partial \Omega_{1} \\ y_{2}(0, \cdot)=y_{0,2}(\cdot), & \text { in } \Omega_{2},\end{cases}
$$

satisfies $y_{2}(T)=0$ in $\Omega_{2}$.

This is in contradiction with Müntz-Szász theorem when $d \geqslant 2$. Indeed, since the Laplace operator $A=-\Delta$ in $\Omega_{2}$ in $L^{2}\left(\Omega_{2}\right)$ with domain $H^{2} \cap H_{0}^{1}\left(\Omega_{2}\right)$ is positive self-adjoint, its spectrum is formed by a sequence of eigenvalues $\left(\lambda_{k}\right)_{k \in \mathbb{N}}$ going to infinity, and of orthogonal eigenvectors $\left(w_{k}\right)_{k \in \mathbb{N}}$. Besides, according to Weyl's law, the sequence $\left(\lambda_{k}\right)_{k \in \mathbb{N}}$ is equivalent to $C(\Omega) k^{2 / d}$ (see [33], Cor. 4.2, Chap. 13), entailing in particular that $\sum_{k} 1 / \lambda_{k}=\infty$ when $d \geqslant 2$. According to Müntz-Szász theorem (see e.g. [32], p. 91), the set $\left(t \mapsto \exp \left(\sigma_{2} \lambda_{k} t\right)\right)_{k \in \mathbb{N}}$ is complete in $C^{0}([0, T])$, and this is still true even when removing a finite number of elements, for instance its first one. Accordingly there is no solution $Y \in L^{2}(0, T)$ of the moment problem

$$
\int_{0}^{T} Y(t) \exp \left(\sigma_{2} \lambda_{k}(t-T)\right) \mathrm{d} t\left(\int_{\partial \Omega_{1}} \partial_{\nu} w_{k} \mathrm{~d} \gamma\right)=\mathbb{1}_{k=1}, \quad k \in \mathbb{N}
$$

which is the moment problem corresponding to the null-controllability problem for (4.14) with the initial datum $w_{1}$.

\subsection{The case of a strongly insulating material $\sigma_{1} \rightarrow 0$}

It is also interesting to briefly discuss the case $\sigma_{1} \rightarrow 0$ as it corresponds to the limit of a strongly insulating material.

To better understand what happens in this case, let us first present the following convergence result, whose proof is left to the reader:

Proposition 4.4. Let $\Omega$ be a smooth $\left(\mathscr{C}^{2}\right)$ bounded domain in $\mathbb{R}^{d}\left(d \in \mathbb{N}^{*}\right), \Omega_{1} \Subset \Omega$ be a smooth bounded domain, and $\Omega_{2}=\Omega \backslash \bar{\Omega}_{1}$.

Let $\sigma_{2}>0$ be fixed, $T>0, y_{0} \in L^{2}(\Omega)$, and let $\left(f_{\sigma}\right)_{\sigma_{1}>0}$ be a bounded family of $L^{2}\left(0, T ; L^{2}(\Omega)\right)$, which weakly converges as $\sigma_{1} \rightarrow 0$ to $f^{*}$ in $L^{2}\left(0, T ; L^{2}(\Omega)\right)$. 
For $\sigma_{1}>0$, and $\sigma$ as in (1.1), let $\left(y_{\sigma}\right)_{\sigma_{1}>0}$ be the solution of

$$
\begin{cases}\partial_{t} y_{\sigma}-\operatorname{div}\left(\sigma \nabla y_{\sigma}\right)=f_{\sigma}, & \text { in }(0, T) \times \Omega, \\ y_{\sigma}=0, & \text { on }(0, T) \times \partial \Omega, \\ y_{\sigma}(0)=y_{0}, & \text { in } \Omega .\end{cases}
$$

Then as $\sigma_{1} \rightarrow 0$, the sequence $\left(y_{\sigma}\right)_{\sigma_{1}>0}$ weakly converges in $L^{2}\left(0, T ; L^{2}(\Omega)\right)$ to the solution $y^{*}$ of

$$
\begin{cases}\partial_{t} y^{*}-\operatorname{div}\left(\mathbb{1}_{\Omega_{2}} \sigma_{2} \nabla y^{*}\right)=f^{*}, & \text { in }(0, T) \times \Omega, \\ y^{*}=0, & \text { on }(0, T) \times \partial \Omega \\ y^{*}(0)=y_{0}, & \text { in } \Omega\end{cases}
$$

It is then clear that if $\Omega_{1} \backslash \bar{\omega} \neq \emptyset$, the equation (4.16) will not be null-controllable with null-controls localized in the set $\omega$, since in $\Omega_{1} \backslash \bar{\omega}$, the equation (4.16) would then reduce to

$$
\partial_{t} y^{*}=0 \quad \text { in } \mathscr{D}^{\prime}\left((0, T) \times\left(\Omega_{1} \backslash \bar{\omega}\right)\right) .
$$

Still, an interesting open question is the analysis of the rate at which the cost of controllability blows up as $\sigma_{1}$ goes to 0.

Acknowledgements. The authors are indebted to Alberto Mercado for having suggested the simple proof by multipliers of Theorem 1.4, to Mehdi Badra for having pointed out the reference [28], and to the anonymous referee for having pointed out the work [18].

\section{REFERENCES}

[1] G. Alessandrini and L. Escauriaza, Null-controllability of one-dimensional parabolic equations. ESAIM: COCV 14 (2008) 284-293.

[2] C. Bardos, G. Lebeau and J. Rauch, Sharp sufficient conditions for the observation, control and stabilization of waves from the boundary. SIAM J. Control Optim. 30 (1992) 1024-1065.

[3] L. Baudouin and A. Mercado, An inverse problem for Schrödinger equations with discontinuous main coefficient. Appl. Anal. 87 (2008) 1145-1165.

[4] L. Baudouin, A. Mercado and A. Osses, A global Carleman estimate in a transmission wave equation and application to a one-measurement inverse problem. Inverse Prob. 23 (2007) 257.

[5] A. Benabdallah, Y. Dermenjian and J. Le Rousseau, Carleman estimates for the one-dimensional heat equation with a discontinuous coefficient and applications to controllability and an inverse problem. J. Math. Anal. Appl. 336 (2007) 865-887.

[6] A. Benabdallah, Y. Dermenjian and J. Le Rousseau, Carleman estimates for stratified media. J. Funct. Anal. 260 (2011) $3645-3677$.

[7] A Benabdallah, Y. Dermenjian and L. Thevenet, Carleman estimates for some non-smooth anisotropic media. Commun. Partial Differ. Equ. 38 (2013) 1763-1790.

[8] F. Boyer, Controllability of linear parabolic equations and systems, Lecture (2020), https://hal.archives-ouvertes.fr/ hal-02470625.

[9] B. Dehman and S. Ervedoza, Observability estimates for the wave equation with rough coefficients. C. R. Math. Acad. Sci. Paris 355 (2017) 499-514.

[10] A. Doubova, A. Osses and J.-P. Puel, Vol. 8 of Exact controllability to trajectories for semilinear heat equations with discontinuous diffusion coefficients. A tribute to J. L. Lions (2002) 621-661.

[11] S. Ervedoza and E. Zuazua, Sharp observability estimates for heat equations. Arch. Ratl. Mech. Anal. 202 (2011) 975-1017.

[12] S. Ervedoza and E. Zuazua, Observability of heat processes by transmutation without geometric restrictions. Math. Control Relat. Fields 1 (2011) 177-187.

[13] L.C. Evans, Partial differential equations, Vol. 19 of Graduate Studies in Mathematics. American Mathematical Society, Providence, RI (1998).

[14] H.O. Fattorini and D.L. Russell, Exact controllability theorems for linear parabolic equations in one space dimension. Arch. Ratl. Mech. Anal. 43 (1971) 272-292.

[15] A.V. Fursikov and O.Y. Imanuvilov, Controllability of evolution equations. Vol. 34 of Lecture Notes Series. Seoul National University Research Institute of Mathematics Global Analysis Research Center, Seoul (1996). 
[16] L. Gagnon, Sufficient conditions for the controllability of wave equations with a transmission condition at the interface. arXiv preprint arXiv:1711.00448 (2017).

[17] L.F. Ho, Observabilité frontière de l'équation des ondes. C. R. Acad. Sci. Paris Sér. I Math. 302 (1986) $443-446$.

[18] A. Khoutaibi, Contrôlabilité à zéro d'une classe d'équations paraboliques linéaires et semi-linéaires avec des conditions aux limites dynamiques, Ph.D. Thesis, Univ. Cadi Ayyad (2020).

[19] J. Le Rousseau, Carleman estimates and controllability results for the one-dimensional heat equation with BV coefficients. $J$. Differ. Equ. 233 (2007) 417-447.

[20] J. Le Rousseau and N. Lerner, Carleman estimates for anisotropic elliptic operators with jumps at an interface. Anal. PDE 6 (2013) 1601-1648.

[21] J. Le Rousseau and L. Robbiano, Carleman estimate for elliptic operators with coefficients with jumps at an interface in arbitrary dimension and application to the null controllability of linear parabolic equations. Arch. Ratl. Mech. Anal. 195 (2010) 953-990.

[22] J. Le Rousseau and L. Robbiano, Local and global Carleman estimates for parabolic operators with coefficients with jumps at interfaces. Invent. Math. 183 (2011) 245-336.

[23] G. Lebeau and L. Robbiano, Contrôle exact de l'équation de la chaleur. Comm. Partial Differ. Equ. 20 (1995) $335-356$.

[24] J.-L. Lions, Contrôlabilité exacte, perturbations et stabilisation de systèmes distribués. Tome 1 , vol. 8 of Recherches en Mathématiques Appliquées. Contrôlabilité exacte, With appendices by E. Zuazua, C. Bardos, G. Lebeau and J. Rauch. Masson, Paris (1988).

[25] P. Martin, L. Rosier and P. Rouchon, Null controllability of one-dimensional parabolic equations by the flatness approach. SIAM J. Control Optim. 1 (2016) 198-220.

[26] L. Miller, Controllability cost of conservative systems: resolvent condition and transmutation. J. Funct. Anal. 218 (2005) 425-444.

[27] L. Miller, The control transmutation method and the cost of fast controls. SIAM J. Control Optim. 45 (2006) $762-772$.

[28] J.-P. Raymond and M. Vanninathan, Null controllability in a heat-solid structure model. Appl. Math. Optim. 59 (2009) $247-273$.

[29] D.L. Russell, A unified boundary controllability theory for hyperbolic and parabolic partial differential equations. Stud. Appl. Math. 52 (1973) 189-211.

[30] D.L. Russell, Controllability and stabilizability theory for linear partial differential equations: recent progress and open questions. SIAM Rev. 20 (1978) 639-739.

[31] M. Tucsnak and G. Weiss. Observation and control for operator semigroups, Birkhäuser Advanced Texts: Basler Lehrbücher. [Birkhäuser Advanced Texts: Basel Textbooks]. Birkhäuser Verlag, Basel (2009).

[32] R.M. Young, An introduction to nonharmonic Fourier series, Academic Press Inc., San Diego, CA, first edition (2001).

[33] C. Zuily, Éléments de distributions et d'Équations aux dérivées Partielles. Dunod, Sciences Sup (2002). 